\title{
Kinematic Modeling and Optimization of a New Reconfigurable Parallel Mechanism
}

\author{
Wei Ye ${ }^{1,2 *}$, Xinxue Chai $^{1}$, Ketao Zhang ${ }^{2 *}$ \\ 1 Faculty of Mechanical Engineering and Automation, Zhejiang Sci-Tech University, Hangzhou, 310018, P.R.China \\ 2 School of Engineering and Material Science, Queen Mary University of London, London, E1 4NS, UK
}

\begin{abstract}
This paper investigates a new reconfigurable parallel mechanism consisting of three $\mathrm{S}_{\mathrm{v}} \mathrm{PS}$ kinematic limbs. Induced by phase changes of a metamorphic spherical variable-axis joint $\left(S_{v}\right)$, the $S_{v} P S$ limb is capable of changing to two typical configurations, of which one exerts no constraint and the other exerts a constraint force to the moving platform. Reconfiguration of the three limbs enables the 3-S $\mathrm{S}_{\mathrm{v}} \mathrm{PS}$ parallel mechanism to have four distinct configurations with degrees of freedom (DOF) varying from 3 to 6 . Analytical model of position and workspace analysis of the reconfigurable parallel mechanism are first investigated. In terms of the screw theory, a unified Jacobian matrix covering all the mobility configurations is established and utilized for analyzing singularity of the parallel mechanism in different configurations. Further, performance analysis and optimization are explored using the motion/force transmission method. The 3-S PS parallel mechanism can be used as structure of reconfigurable robotic machine center with adaptability to changing task requirements.
\end{abstract}

KEYWORDS: Reconfigurable parallel mechanism, Unified Jacobian matrix, Performance analysis, Adaptability.

\section{Introduction}

Compared with serial mechanisms with open loop structures, parallel mechanisms with a moving platform connected to a fixed base by at least two kinematic limbs have multi-loop structures, whose stiffness is inherently excellent. Furthermore, the multi-loop structure makes it possible to mount all the actuations near to the fixed base, which reduces the moving mass and results in good dynamic performance. In view of these advantages, parallel mechanisms have been extensively studied in the past three decades. A lot of robotic equipment were successfully developed based on parallel mechanisms [1-6].

Traditional parallel mechanisms have constant topological configurations and fixed mobility, which limit their versatility and adaptability. Inventing mechanisms with reconfigurability and adaptability to meet multi-task requirements in modern automation field [7] became a new trend. In this context, a lot of works about reconfigurable parallel mechanisms (RPMs) were carried out and reported in recent years. Reconfigure strategies in existing RPMs mainly include using constraint singularities [8-13], introducing metamorphic or lockable joints [14-23], and integrating in kinematic limbs some reconfigurable linkages [24-26]. Kong et al. synthesized RPMs with multiple operation modes using the screw-based method [8-10]. Li et al. [12] presented a class of RPMs with bifurcation of Schoenflies motion using the displacement group-based method. Gogu [13] presented 3-DOFs (degrees of freedom) RPMs that can perform rotations around different axes in different configurations. Those RPMs can evolve into different operation modes from constraint singular configurations [27], in which the mobility is instantaneously increased. Gan et al. [14-17] invented the metamorphic rT joint, $\mathrm{rR}$ joint, and presented an approach for construction of RPMs using those joints. Zhang et al. [18-19] presented a vA (variable-Axis) joint inspired by origami and integrated it in the design of several RPMs. Carbonari et al. [20-22] designed a class of RPMs by introducing a special lockable universal joint. Phase changes in those metamorphic or lockable joints result in mobility reconfiguration of the associated RPMs. Finistauri et al. [23] utilized lockable universal-to-revolute joint to construct a RPM with variable mobility from 1 to 6. Ye et al. [24-26] presented several kinds of RPMs by integrating reconfigurable linkages in kinematic limbs. Mobility variation of those mechanisms can be achieved through configuration changes of the reconfigurable linkages.

Structural design and kinematics performance are two important topics in researches of mechanisms. The former is to invent novel mechanical structures for potential applications, and the latter is to know the performance of the proposed mechanisms and optimize it if necessary, so as to better accommodate the task. Of note is that these work about RPMs were mainly focused on structural design. Kinematics modeling and performance analysis are confined to the rT, rR joint-related RPMs [17, 28-30] and the lockable universal joint-related RPMs [21,31]. This paper presents a novel RPM based on a vA joint, and investigates its kinematics, performance and optimization. Induced by phase changes of the vA joint, the RPM can switch between 6-DOF, 5DOF, 4-DOF and 3-DOF configurations. It has promising potential to be used for constructing reconfigurable machine center to provide various solutions for part machining. Remainder of the paper is arranged as follows: Section 2 introduces the novel RPM and analyzes its reconfigurability. This is following by kinematic, workspace, Jacobian and singularity analysis in section 3 . Section 4 reports performance analysis and optimization for the proposed RPM, and section 5 concludes the work.

*Corresponding authors (email: wye@zstu.edu.cn, ketao.zhang@qmul.ac.uk) 


\section{A new reconfigurable parallel mechanism}

The vA joint is a reconfigurable joint extracted from an L-shaped cardboard [18]. As in Fig.1(a), the joint has three rotational DOFs in a general configuration, which is called the source phase $\left(S_{v}\right)$. The three rotational axes are denoted $R_{1}, R_{2}$, and $R_{3}$, respectively. The angles between axis $R_{1}$ and $R_{2}, R_{2}$ and $R_{3}$ are $45 \mathrm{deg}$. When the joint move to the configuration with link 2 annexed with link 1 and axis $R_{3}$ perpendicular to axis $R_{1}$ as in Fig.1(b), the vA joint can be regarded as an equivalent Hooke's joint in which link 3 has only two rotational DOFs with respect to link 0 , which is denoted by $\mathrm{U}_{\mathrm{v}}$ phase. When the joint change its configuration to a phase with link 2 annexed with link 1 and axis $R_{3}$ coincident with axis $R_{1}$ as in Fig.1(c), the joint can be taken as a revolute joint, which is denoted by $\mathrm{R}_{\mathrm{v}}$ phase. Denote the rotational angles of $\mathrm{R}_{1}, \mathrm{R}_{2}$, and $\mathrm{R}_{3}$ using $\theta$, $\delta$, and $\psi$, respectively. When the joint is in the $\mathrm{U}_{\mathrm{v}}$ phase, $\delta$ is fixed as $180 \mathrm{deg}$. There are only two angle variables $\theta$ and $\psi$ in this phase. When the joint is in the $\mathrm{R}_{\mathrm{v}}$ phase, $\delta$ is fixed as 0 , and there are only one angle variable $\theta$ because the axes of $\mathrm{R}_{1}$ and $\mathrm{R}_{3}$ are coincident. Three phases of the vA joint correspond to the three morphologies of the L-shaped cardboard. The vA joint can switch between different phases by annexing/releasing links. It has been used to construct several reconfigurable parallel mechanisms [18-19]. Table.1 summarizes the features of the vA joint in different phases.

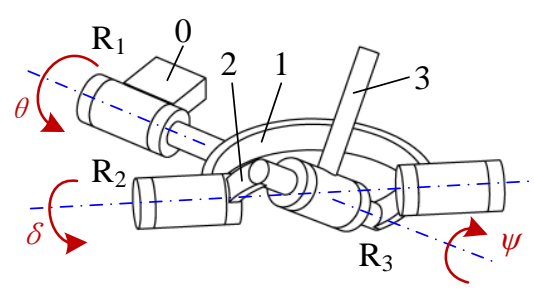

(a)

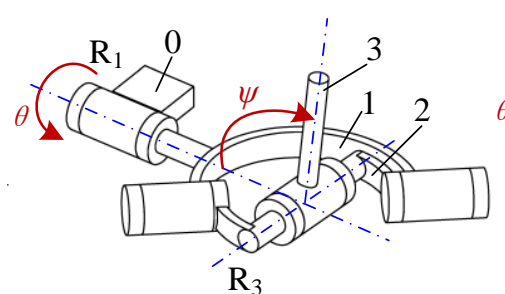

(b)

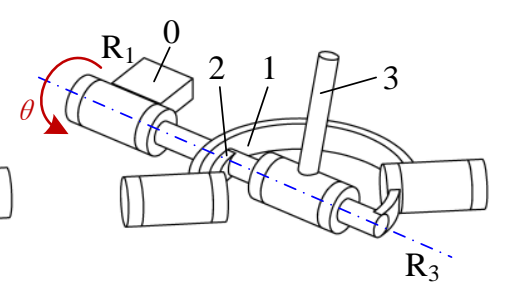

(c)

Fig.1 The vA joint: (a) source phase $S_{v}$, (b) $U_{v}$ phase, (c) $R_{v}$ phase

Table.1 Three phases of the vA joint

\begin{tabular}{cccc}
\hline Different phases & Source phase $\mathrm{Sv}$ & Uv phase & $\mathrm{Rv}$ phase \\
\hline Annexed links & None & Links 1 and 2 & Links 1 and 2 \\
Geometrical conditions & ${\text { Angles between } \mathrm{R}_{1} \text { and } \mathrm{R}_{2},}_{\mathrm{R}_{2} \text { and } \mathrm{R}_{3} \text {, are } 45 \mathrm{deg}}^{\text {Axes } \mathrm{R}_{1} \text { and } \mathrm{R}_{3} \text { are }}$ & perpendicular & Axes $\mathrm{R}_{1}$ and $\mathrm{R}_{3}$ are \\
Joint variables & $\theta, \delta$ and $\psi$ & $\theta$ and $\psi$ & coincident \\
\hline
\end{tabular}

Integrating the vA joint in the structure design of serial kinematic chains, parallel mechanisms that have reconfigurability can be constructed. Fig. 2 shows a novel parallel mechanism formed by connecting a moving platform and a fixed base through three identical kinematic limbs. Counted form the fixed base to the moving platform, each limb contains a vA joint, a P (prismatic) joint, a $S$ (spherical) joint, and two links connecting these joints. Induced by phase changes of the vA joint, this parallel mechanism is capable of switching between different mobility configurations. It will be denoted by $3 S_{\mathrm{v}} \mathrm{PS}$ RPM (reconfigurable parallel mechanism) hereinafter.

Corresponding to three phases of the vA joint, the serial kinematic limb $S_{\mathrm{v}} \mathrm{PS}$ also has three configurations, i.e., the SPS configuration with the vA joint in its source phase, the $U_{v} P S$ configuration with the vA joint in its $U_{v}$ phase, and the $R_{v} P S$ configuration with the $\mathrm{vA}$ joint in its $\mathrm{R}_{\mathrm{v}}$ phase. Axis of the revolute joint and that of the prismatic joint in the $\mathrm{R}_{\mathrm{v}} \mathrm{PS}$ configuration are designed to be perpendicular to each other. It should be noted that the SPS configuration has a local mobility that allows the $\mathrm{P}$ joint rotating around a line passing through the centers of the two S joints. Replacing SPS limbs in a parallel mechanism by UPS limbs will not affect its mobility. Therefore, only the $U_{v} P S$ configuration and the $R_{v} P S$ configuration of the $S_{v} P S$ limb are considered in this paper. The $3 S_{v} P S$ RPM has four distinct configurations, i.e., the $3 U_{v} P S$ configuration, the $2 U_{v} P S-1 R_{v} P S$ configuration, the $1 \mathrm{U}_{\mathrm{v}} \mathrm{PS}-2 \mathrm{R}_{\mathrm{v}} \mathrm{PS}$ configuration, and the $3 \mathrm{R}_{\mathrm{v}} \mathrm{PS}$ configuration, as shown in Fig. 2 .

The $3 U_{v} P S$ configuration has 6 DOFs since the $U_{v} P S$ limbs exert no constraint on the moving platform. While the $R_{v} P S$ limb provides a constraint force that is parallel to the axis of the $R_{v}$ joint and passes through the center of the $S$ joint. Such a constraint restricts the translational motion of the platform along the direction of the constraint force. Therefore, the $2 U_{v} P S-1 R_{v} P S$ configuration losses a translational motion and implements $3 \mathrm{R} 2 \mathrm{~T}$ (R: rotation, $\mathrm{T}$ : translation) motion. The $1 \mathrm{U}_{\mathrm{v}} \mathrm{PS}-2 \mathrm{R}_{\mathrm{v}} \mathrm{PS}$ configuration losses two translational motion and implements $3 \mathrm{R} 1 \mathrm{~T}$ motion. The $3 \mathrm{R}_{\mathrm{v}} \mathrm{PS}$ configuration implements $2 \mathrm{R} 1 \mathrm{~T}$ motion [32] since all the three constraint forces are parallel to a same plane that restrict two translational motion and a rotational motion in that plane.

Since the $3 \mathrm{~S}_{\mathrm{v}} \mathrm{PS}$ parallel mechanism has a maximum of 6 DOFs and only three kinematic limbs, each limb should have two actuations. We can choose the revolute joint $\mathrm{R}_{1}$ in the $\mathrm{vA}$ joint and the prismatic joint in each limb as input joints. Note that the two input joints are both active when the limb is in its $\mathrm{U}_{\mathrm{v}} \mathrm{PS}$ configuration. While the limb is in the $\mathrm{R}_{\mathrm{v}} \mathrm{PS}$ configuration, only the prismatic joint is active and $R_{1}$ becomes a passive joint. In this way, the number of inputs equals to the DOFs in all the configurations. Motion of the RPM can be fully controlled with no redundant actuations through this strategy. 
To switch the vAjoint between the $U_{v}$ phase and the $R_{v}$ phase as required in the presented RPM, we can equip joint $R_{2}$ with a rotary motor and a worm gear transmission with self-locking characteristic, or a rotary motor with brake system. Other physical locking devices such as bolts or stoppers can also be added to reinforce the locking stability.
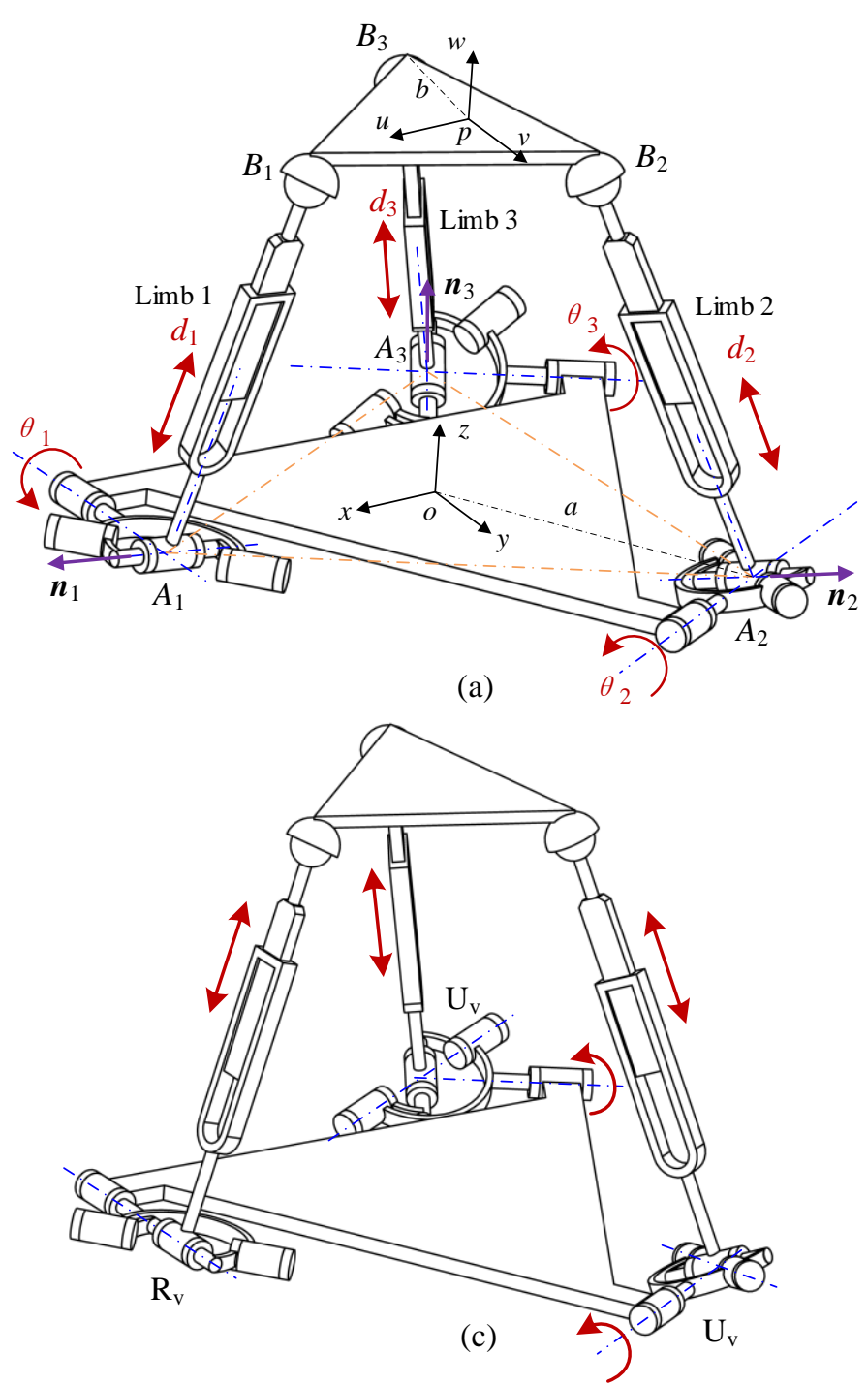

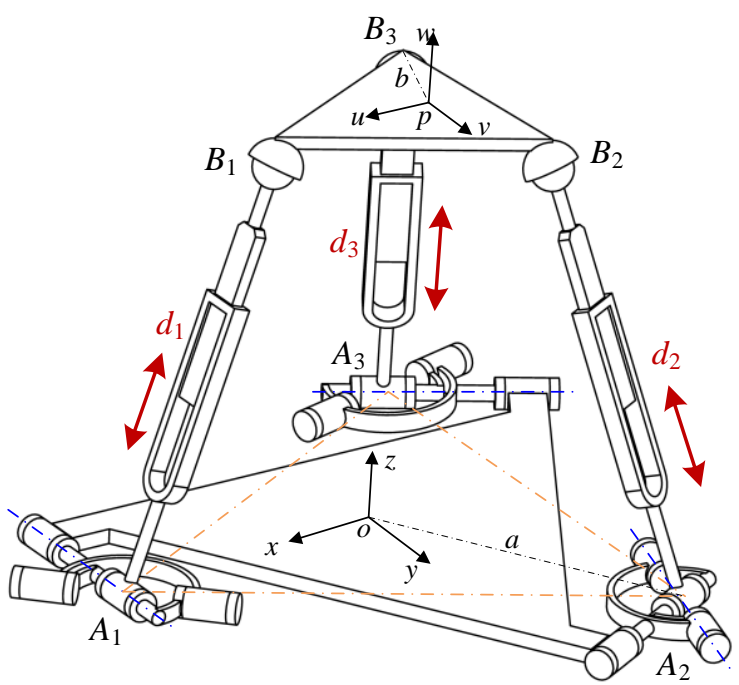

(b)

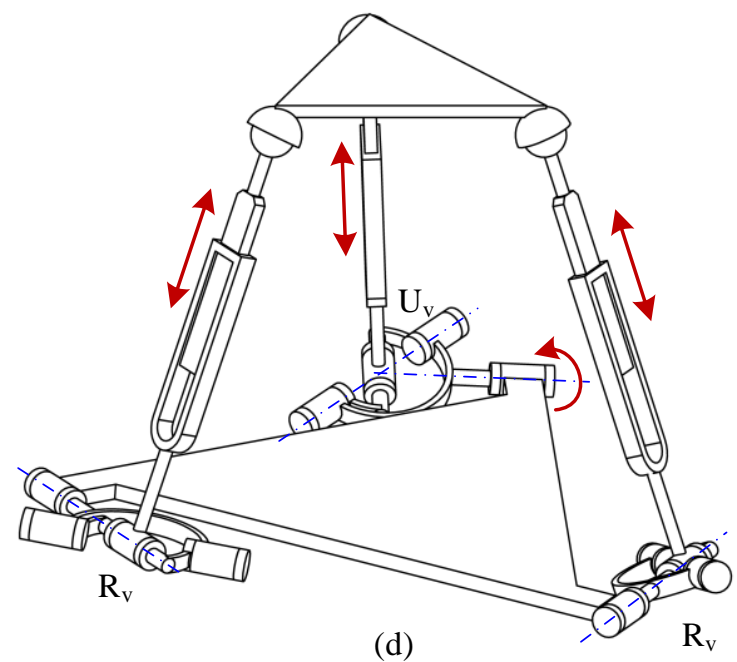

Fig.2 Four configurations of the $3 S_{v} P S$ RPM: (a) the $3 U_{v} P S$ configuration, (b) the $3 R_{v} P S$ configuration, (c) the $2 U_{v} P S-1 R_{v} P S$ configuration, (d) the $1 \mathrm{U}_{\mathrm{v}} \mathrm{PS}-2 \mathrm{R}_{\mathrm{v}} \mathrm{PS}$ configuration

The various mobility configurations of the RPM increase its adaptability in tasks with multi-stages. Fig. 3 shows a concept of a reconfigurable machine center (RMC) formed by mounting the RPM to a X-Y gantry. This concept is similar to the famous machine Ecospeed [2], which is used in manufacture of aluminum structures. Of note is that when the Ecospeed is working, the two translational motion of the X-Y gantry are both required, which consumes a lot of energy since the heavy parallel module is mounted on the X-Y gantry and the gantry should be actuated by powerful motors. While the RMC would provide different solutions: when the workpiece is small, the RPM can reconfigure to the 6-DOF configuration or 5-DOF configuration to perform the task, in which the X-Y gantry doesn't need to be actuated. When the workpiece is large, it can be divided into small portions, and the RPM is adjusted to different positions on the gantry to finish different portions, which implies the gantry is working only when the RPM needs to move from one position to another, and can be fixed once the targeted position is reached. Certainly, the RMC can also deal with large workpiece like the Ecospeed by reconfiguring the RPM to the 3-DOF configuration and actuating translational motion of the gantry.

It is worth mentioning we can obtain three 5-DOF configurations from the 6-DOF configuration by reconfiguring different limbs. Although the three 5-DOF configurations have the same topological structure, their performance distribution in the workspace will be different once the RMC is installed. It means that the RMC can reconfigure its performance in the same mobility configurations to better finish its task. 


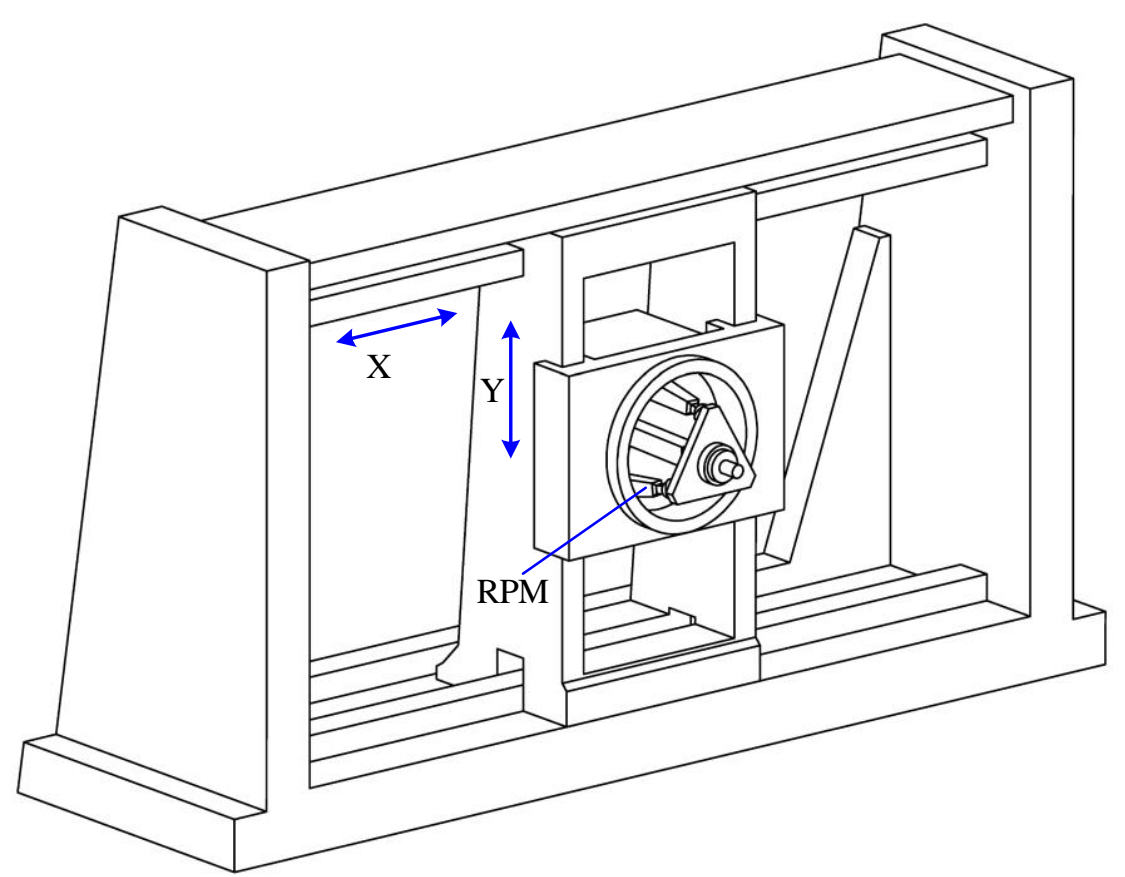

Fig.3 A reconfigurable machine center based on the RPM

\section{Kinematics analysis}

Although the $3 \mathrm{~S}_{\mathrm{v}} \mathrm{PS}$ RPM has four mobility configurations, here we establish a unified kinematics model since the configurations have internal connections and can switch between each other. Firstly, geometrical conditions in the RPM will be introduced. As in Fig. 2(a-b), the three vA joints are symmetrically located on the fixed base plane. Center $A_{1}, A_{2}$ and $A_{3}$ of the three vA joints form an equilateral triangle whose circumradius is $a$. Axes of the three $\mathrm{R}_{1}$ joints also form an equilateral triangle. Centers $B_{1}, B_{2}$ and $B_{3}$ of the three spherical joints connected to the moving platform are symmetrically located on a circle with radius $b$.

\subsection{Position modeling}

Position analysis aims to establish the mapping between input variables and output parameters. As aforementioned, the $\mathrm{S}_{\mathrm{v}} \mathrm{PS}$ limb in the $\mathrm{U}_{\mathrm{v}} \mathrm{PS}$ configuration has two input joints. One is the first revolute joint in the vA joint and the other is the P joint. Denote the rotational input variable in limb $i$ as $\theta_{i}$, and the translational input variable as $d_{i}(i=1,2,3)$. While when the limb is in the $\mathrm{R}_{\mathrm{v}} \mathrm{PS}$ configuration, the first and third rotational axes of the vA joint are coincident. The limb has only one input $d_{i}$ in that configuration. Attach a global coordinate system $G(o-x y z)$ to the fixed base with origin $o$ located at the center of the base. The $x$ axis passes through point $A_{1}$ while $z$-axis is perpendicular to the base plane, and $y$-axis is defined according to the right-hand rule. Similarly, a local coordinate system $L(p-u v w)$ is attached to the moving platform with its origin $p$ located at the center of the platform, $u$-axis passing through point $B_{1}, w$-axis perpendicular to the platform plane, and $v$-axis defined according to the righthand rule.

Position of the moving platform can be expressed using the vector $\boldsymbol{p}=[x, y, z]^{\mathrm{T}}$ of the origin of the local coordinate system. To express the orientation of the moving platform with respect to the fixed base, the $z-y-x$ Euler angles are used here. The rotation matrix is expressed as

$$
{ }^{G} \boldsymbol{R}_{L}=\boldsymbol{R}_{x y z}(\gamma, \quad \beta, \alpha)=\left[\begin{array}{ccc}
\mathrm{c} \alpha \mathrm{c} \beta & \mathrm{c} \alpha \mathrm{s} \beta \mathrm{s} \gamma-\mathrm{s} \alpha \mathrm{c} \gamma & \mathrm{c} \alpha \mathrm{s} \beta \mathrm{c} \gamma+\mathrm{s} \alpha \mathrm{s} \gamma \\
\mathrm{s} \alpha \mathrm{c} \beta & \mathrm{s} \alpha \mathrm{s} \beta \mathrm{s} \gamma+\mathrm{c} \alpha \mathrm{c} \gamma & \mathrm{s} \alpha \mathrm{s} \beta \mathrm{c} \gamma-\mathrm{c} \alpha \mathrm{s} \gamma \\
-\mathrm{s} \beta & \mathrm{c} \beta \mathrm{s} \gamma & \mathrm{c} \beta \mathrm{c} \gamma
\end{array}\right]
$$

where $\mathrm{c} *$ and $\mathrm{s} *$ denote $\cos (*)$ and $\sin (*)$, respectively.

Position vectors of points $A_{i}(i=1,2,3)$ in the global coordinate system can be expressed as

$$
\boldsymbol{A}_{1}=[a, 0,0]^{\mathrm{T}}, \boldsymbol{A}_{2}=\left[-\frac{a}{2}, \frac{\sqrt{3} a}{2}, 0\right]^{\mathrm{T}}, \boldsymbol{A}_{3}=\left[-\frac{a}{2},-\frac{\sqrt{3} a}{2}, 0\right]^{\mathrm{T}}
$$

With respect to the local coordinate system, position vectors of points $B_{i}(i=1,2,3)$ can be expressed as

$$
{ }^{L} \boldsymbol{B}_{1}=[b, 0,0]^{\mathrm{T}},{ }^{L} \boldsymbol{B}_{2}=\left[-\frac{b}{2}, \frac{\sqrt{3} b}{2}, 0\right]^{\mathrm{T}},{ }^{L} \boldsymbol{B}_{3}=\left[-\frac{b}{2},-\frac{\sqrt{3} b}{2}, 0\right]^{\mathrm{T}}
$$

where the left superscript $L$ denotes that the reference coordinate system is the local one.

Position vectors of points $B_{i}$ expressed in the global coordinate system can be given by 
Substituting Eqs. (1) and (3) into Eq.(4) yields

$$
\boldsymbol{B}_{i}={ }^{G} \boldsymbol{R}_{L} \cdot{ }^{L} \boldsymbol{B}_{i}+\boldsymbol{P}
$$

$$
\begin{aligned}
& \boldsymbol{B}_{1}=[x+b \mathrm{c} \alpha \mathrm{c} \beta, y+b \mathrm{~s} \alpha \mathrm{c} \beta, z-b \mathrm{~s} \beta]^{\mathrm{T}} \\
& \boldsymbol{B}_{2}=\left[x-\frac{b}{2} \mathrm{c} \alpha \mathrm{c} \beta+\frac{\sqrt{3} b}{2}(\mathrm{c} \alpha \mathrm{s} \beta \mathrm{s} \gamma-\mathrm{s} \alpha \mathrm{c} \gamma), y-\frac{b}{2} \mathrm{~s} \alpha \mathrm{c} \beta+\frac{\sqrt{3} b}{2}(\mathrm{~s} \alpha \mathrm{s} \beta \mathrm{s} \gamma+\mathrm{c} \alpha \mathrm{c} \gamma), z+\frac{b}{2} \mathrm{~s} \beta+\frac{\sqrt{3} b}{2} \mathrm{c} \beta \mathrm{s} \gamma\right]^{\mathrm{T}} \\
& \boldsymbol{B}_{3}=\left[x-\frac{b}{2} \mathrm{c} \alpha \mathrm{c} \beta-\frac{\sqrt{3} b}{2}(\mathrm{c} \alpha \mathrm{s} \beta \mathrm{s} \gamma-\mathrm{s} \alpha \mathrm{c} \gamma), y-\frac{b}{2} \mathrm{~s} \alpha \mathrm{c} \beta-\frac{\sqrt{3} b}{2}(\mathrm{~s} \alpha \mathrm{s} \beta \mathrm{s} \gamma+\mathrm{c} \alpha \mathrm{c} \gamma), z+\frac{b}{2} \mathrm{~s} \beta-\frac{\sqrt{3} b}{2} \mathrm{c} \beta \mathrm{s} \gamma\right]^{\mathrm{T}}
\end{aligned}
$$

A constraint equation can be established for limb $i$ as

$$
\left|\boldsymbol{A}_{i} \boldsymbol{B}_{i}\right|=d_{i} \quad(i=1,2,3)
$$

where $\boldsymbol{A}_{i} \boldsymbol{B}_{i}=\boldsymbol{B}_{i}-\boldsymbol{A}_{i}$, and $d_{i}$ is the length of vector $\boldsymbol{A}_{i} \boldsymbol{B}_{i}$.

Substituting Eqs. (2) and (5) into Eq.(6) yields

$$
\begin{gathered}
\sqrt{(x+b \mathrm{c} \alpha \mathrm{c} \beta-a)^{2}+(y+b \mathrm{~s} \alpha \mathrm{c} \beta)^{2}+(z-b \mathrm{~s} \beta)^{2}}=d_{1} \\
\sqrt{\left(x-\frac{b}{2} \mathrm{c} \alpha \mathrm{c} \beta+\frac{\sqrt{3} b}{2}(\mathrm{c} \alpha \mathrm{s} \beta \mathrm{s} \gamma-\mathrm{s} \alpha \mathrm{c} \gamma)+\frac{a}{2}\right)^{2}+\left(y-\frac{b}{2} \mathrm{~s} \alpha \mathrm{c} \beta+\frac{\sqrt{3} b}{2}(\mathrm{~s} \alpha \mathrm{s} \beta \mathrm{s} \gamma+\mathrm{c} \alpha \mathrm{c} \gamma)-\frac{\sqrt{3} a}{2}\right)^{2}+\left(z+\frac{b}{2} \mathrm{~s} \beta+\frac{\sqrt{3} b}{2} \mathrm{c} \beta \mathrm{s} \gamma\right)^{2}}=d_{2} \\
\sqrt{\left(x-\frac{b}{2} \mathrm{c} \alpha \mathrm{c} \beta-\frac{\sqrt{3} b}{2}(\mathrm{c} \alpha \mathrm{s} \beta \mathrm{s} \gamma-\mathrm{s} \alpha \mathrm{c} \gamma)+\frac{a}{2}\right)^{2}+\left(y-\frac{b}{2} \mathrm{~s} \alpha \mathrm{c} \beta-\frac{\sqrt{3} b}{2}(\mathrm{~s} \alpha \mathrm{s} \beta \mathrm{s} \gamma+\mathrm{c} \alpha \mathrm{c} \gamma)+\frac{\sqrt{3} a}{2}\right)^{2}+\left(z+\frac{b}{2} \mathrm{~s} \beta-\frac{\sqrt{3} b}{2} \mathrm{c} \beta \mathrm{s} \gamma\right)^{2}}=d_{3}
\end{gathered}
$$

Of note is that the above kinematics analysis is applicable to the RPM in its four configurations since the reconfiguration does not affect the above equations. Three constraint equations are obtained as in Eqs. (7-9). However, rotational input variables and several geometrical constraints in some configurations are not considered.

When the RPM is in the $3 \mathrm{U}_{\mathrm{v}} \mathrm{PS}$ configuration as in Fig.3, vectors $\boldsymbol{n}_{1}, \boldsymbol{n}_{2}$, and $\boldsymbol{n}_{3}$ that represent the axes of R in the vA joints are decided by the rotational input variables $\theta_{1}, \theta_{2}$, and $\theta_{3}$. According to the geometrical conditions, we have

$$
\begin{aligned}
& \boldsymbol{n}_{1}=\boldsymbol{R}_{y}\left(\theta_{1}\right)\left[\begin{array}{ll}
1,0,0 & 0
\end{array}\right]^{\mathrm{T}}=\left[\cos \theta_{1}, 0,-\sin \theta_{1}\right]^{\mathrm{T}} \\
& \boldsymbol{n}_{2}=\boldsymbol{R}_{z}\left(\frac{2 \pi}{3}\right) \boldsymbol{R}_{y}\left(\theta_{2}\right)[1,0,0]^{\mathrm{T}}=\left[-\frac{1}{2} \cos \theta_{2}, \frac{\sqrt{3}}{2} \cos \theta_{2},-\sin \theta_{2}\right]^{\mathrm{T}} \\
& \boldsymbol{n}_{3}=\boldsymbol{R}_{z}\left(\frac{4 \pi}{3}\right) \boldsymbol{R}_{y}\left(\theta_{3}\right)\left[\begin{array}{lll}
1,0,0 & 0
\end{array}\right]^{\mathrm{T}}=\left[-\frac{1}{2} \cos \theta_{3},-\frac{\sqrt{3}}{2} \cos \theta_{3},-\sin \theta_{3}\right]^{\mathrm{T}}
\end{aligned}
$$

where $\boldsymbol{R}_{t}(s)$ is a rotation matrix that represents a rotation angle $s$ around axis $t$.

Since vector $\boldsymbol{n}_{i}$ is perpendicular to vector $\boldsymbol{A}_{i} \boldsymbol{B}_{i}$, i.e., $\boldsymbol{A}_{i} \boldsymbol{B}_{i} \cdot \boldsymbol{n}_{i}=0 \quad(i=1,2,3)$, three constraint equations can be obtained as

$$
\begin{aligned}
& \cos \theta_{1}(x+b \mathrm{c} \alpha \mathrm{c} \beta-a)-\sin \theta_{1}(z-b \mathrm{~s} \beta)=0 \\
& -\frac{1}{2} \cos \theta_{2}\left(x-\frac{b}{2} \operatorname{c} \alpha \mathrm{c} \beta+\frac{\sqrt{3} b}{2}(\operatorname{c} \alpha \mathrm{s} \beta \mathrm{s} \gamma-\mathrm{s} \alpha \mathrm{c} \gamma)+\frac{a}{2}\right)+\frac{\sqrt{3}}{2} \cos \theta_{2}\left(y-\frac{b}{2} \mathrm{~s} \alpha \mathrm{c} \beta+\frac{\sqrt{3} b}{2}(\operatorname{s} \alpha \mathrm{s} \beta \mathrm{s} \gamma+\mathrm{c} \alpha \mathrm{c} \gamma)-\frac{\sqrt{3} a}{2}\right) \\
& -\sin \theta_{2}\left(z+\frac{b}{2} \mathrm{~s} \beta+\frac{\sqrt{3} b}{2} \mathrm{c} \beta \mathrm{s} \gamma\right)=0 \\
& -\frac{1}{2} \cos \theta_{3}\left(x-\frac{b}{2} \operatorname{c} \alpha \mathrm{c} \beta-\frac{\sqrt{3} b}{2}(\cos \beta \mathrm{s} \gamma-\mathrm{s} \alpha \mathrm{c} \gamma)+\frac{a}{2}\right)-\frac{\sqrt{3}}{2} \cos \theta_{3}\left(y-\frac{b}{2} \mathrm{~s} \alpha \mathrm{c} \beta-\frac{\sqrt{3} b}{2}(\operatorname{s} \alpha \mathrm{s} \beta \mathrm{s} \gamma+\operatorname{c} \alpha \mathrm{c} \gamma)+\frac{\sqrt{3} a}{2}\right) \\
& -\sin \theta_{3}\left(z+\frac{b}{2} \mathrm{~s} \beta-\frac{\sqrt{3} b}{2} \mathrm{c} \beta \mathrm{s} \gamma\right)=0
\end{aligned}
$$

When the RPM is in the 3UvPS configuration with six DOFs, there are six input variables, i.e., $d_{1}, d_{2}, d_{3}, \theta_{1}, \theta_{2}, \theta_{3}$ and six independent output parameters $x, y, z, \alpha, \beta, \gamma$. The mapping between input variables and output parameters are established in Eqs. (7-9) and (13-15). When the output parameters are given, the input variables $d_{1}, d_{2}$, and $d_{3}$ are given by Eqs. (7-9). While $\theta_{1}, \theta_{2}$, and $\theta_{3}$ can be derived through Eqs. (13-15) as

$$
\theta_{1}=\arctan \frac{x+b \mathrm{c} \alpha \mathrm{c} \beta-a}{z-b \mathrm{~s} \beta}
$$




$$
\begin{aligned}
& \theta_{2}=\arctan \frac{-\left(x-\frac{b}{2} \mathrm{c} \alpha \mathrm{c} \beta+\frac{\sqrt{3} b}{2}(\mathrm{c} \alpha \mathrm{s} \beta \mathrm{s} \gamma-\mathrm{s} \alpha \mathrm{c} \gamma)+\frac{a}{2}\right)+\sqrt{3}\left(y-\frac{b}{2} \mathrm{~s} \alpha \mathrm{c} \beta+\frac{\sqrt{3} b}{2}(\mathrm{~s} \alpha \mathrm{s} \beta \mathrm{s} \gamma+\mathrm{c} \alpha \mathrm{c} \gamma)-\frac{\sqrt{3} a}{2}\right)}{2 z+b \mathrm{~s} \beta+\sqrt{3} b \mathrm{c} \beta \mathrm{s} \gamma} \\
& \theta_{3}=\arctan \frac{-\left(x-\frac{b}{2} \mathrm{c} \alpha \mathrm{c} \beta-\frac{\sqrt{3} b}{2}(\mathrm{c} \alpha \mathrm{s} \beta \mathrm{s} \gamma-\mathrm{s} \alpha \mathrm{c} \gamma)+\frac{a}{2}\right)-\sqrt{3}\left(y-\frac{b}{2} \mathrm{~s} \alpha \mathrm{c} \beta-\frac{\sqrt{3} b}{2}(\mathrm{~s} \alpha \mathrm{s} \beta \mathrm{s} \gamma+\mathrm{c} \alpha \mathrm{c} \gamma)+\frac{\sqrt{3} a}{2}\right)}{2 z+b \mathrm{~s} \beta-\sqrt{3} b \mathrm{c} \beta \mathrm{s} \gamma}
\end{aligned}
$$

When the RPM reconfigure its configuration to the $3 \mathrm{R}_{\mathrm{v}} \mathrm{PS}$ configuration as in Fig.2(b), we have only three input variables $d_{1}$, $d_{2}, d_{3}$. The six output parameters $x, y, z, \alpha, \beta, \gamma$ are dependent since the RPM has only three DOFs now. Inspecting the geometrical structure of the $3 \mathrm{R}_{\mathrm{v}} \mathrm{PS}$ configuration, it shows that three $\mathrm{S}$ joints are constrained in three fixed planes, respectively. Three geometrical constraint equations can be obtained as

$$
\begin{gathered}
B_{1 y}=0 \quad \text { i.e. } \quad y+b \mathrm{~s} \alpha \mathrm{c} \beta=0 \\
B_{2 y}=-\sqrt{3} B_{2 x} \quad \text { i.e. } \quad y-\frac{b}{2} \mathrm{~s} \alpha \mathrm{c} \beta+\frac{\sqrt{3} b}{2}(\mathrm{~s} \alpha \mathrm{s} \beta \mathrm{s} \gamma+\mathrm{c} \alpha \mathrm{c} \gamma)=-\sqrt{3}\left(x-\frac{b}{2} \mathrm{c} \alpha \mathrm{c} \beta+\frac{\sqrt{3} b}{2}(\mathrm{c} \alpha \mathrm{s} \beta \mathrm{s} \gamma-\mathrm{s} \alpha \mathrm{c} \gamma)\right) \\
B_{3 y}=\sqrt{3} B_{3 x} \quad \text { i.e. } \quad y-\frac{b}{2} \mathrm{~s} \alpha \mathrm{c} \beta-\frac{\sqrt{3} b}{2}(\operatorname{s} \alpha \mathrm{s} \beta \mathrm{s} \gamma+\mathrm{c} \alpha \mathrm{c} \gamma)=\sqrt{3}\left(x-\frac{b}{2} \mathrm{c} \alpha \mathrm{c} \beta-\frac{\sqrt{3} b}{2}(\mathrm{c} \alpha \mathrm{s} \beta \mathrm{s} \gamma-\mathrm{s} \alpha \mathrm{c} \gamma)\right)
\end{gathered}
$$

where $B_{i x}$ and $B_{i y}$ are coordinates of point $B_{i}$ in axis $x$ and $y$, respectively.

Eqs. (19-21) mean that only three of the six output parameters in the $3 \mathrm{R}_{\mathrm{v}} \mathrm{PS}$ configuration are independent. Parameters $\beta, \gamma$ and $z$ can be regarded as independent, and the other three parameters are decided by $\beta, \gamma$ and $z$. When three independent output parameters $\beta, \gamma$ and $z$ are given, the input variables $d_{1}, d_{2}, d_{3}$ can be determined using Eqs. (7-9) and (19-21).

RPM in the $2 \mathrm{U}_{\mathrm{v}} \mathrm{PS}-1 \mathrm{R}_{\mathrm{v}} \mathrm{PS}$ configuration has five DOFs and five input variables. Provide that limb 1 is in the $\mathrm{R}_{\mathrm{v}} \mathrm{PS}$ configuration without loss of generality, the five input variables are $d_{1}, d_{2}, d_{3}, \theta_{2}$, and $\theta_{3}$. Output parameters $x, z, \alpha, \beta, \gamma$ can be regarded as independent, and $y$ is calculated using Eq. (19). Inverse solutions for the RPM in this configuration can be derived using Eqs. (7-9) and (17-19).

Similarly, assume that limbs 1 and 2 of the $1 \mathrm{U}_{\mathrm{v}} \mathrm{PS}-2 \mathrm{R}_{\mathrm{v}} \mathrm{PS}$ configuration are $\mathrm{R}_{\mathrm{v}} \mathrm{PS}$ limbs, the four input variables are $d_{1}, d_{2}, d_{3}$, and $\theta_{3}$. Output parameters $z, \alpha, \beta, \gamma$ can be regarded as independent, and $x, y$ is calculated using Eqs. (19) and (20). Inverse solutions for the RPM in this configuration can be derived using Eqs. (7-9) and (18-20).

For clarity, constraints equations and inverse position solutions for the RPM in different configurations are summarized in Table.2.

Table.2 Mathematical equations for the RPM

\begin{tabular}{ccc}
\hline RPM configurations & Constraint equations & Inverse position solutions \\
\hline $3 U_{\mathrm{v}} P S$ & Eqs. (7-9), Eqs. (13-15) & Eqs. (7-9), Eqs. (16-18) \\
$2 \mathrm{U}_{\mathrm{v}} \mathrm{PS}-1 \mathrm{R}_{\mathrm{v}} \mathrm{PS}$ & Eqs. (7-9), Eqs. (14-15), Eq. (19) & Eqs. (7-9), Eqs. (17-18) \\
$1 \mathrm{U}_{\mathrm{v}} \mathrm{PS}-2 \mathrm{R}_{\mathrm{v}} \mathrm{PS}$ & Eqs. (7-9), Eq. (15), Eqs. (19-20) & Eqs. (7-9), Eqs. (18) \\
$3 \mathrm{R}_{\mathrm{v}} \mathrm{PS}$ & Eqs. (7-9), Eqs. (19-21) & Eqs. (7-9) \\
\hline
\end{tabular}

\subsection{Workspace}

Workspace analysis should take into consideration some physical limitations, such as stroke of actuators and link interference, etc. For the $3 S_{\mathrm{v}} \mathrm{PS} R \mathrm{RPM}$, three constraints of the workspace are considered. First, $d_{i}(i=1,2,3)$ is limited in a range of $80 \mathrm{~mm} 220$ $\mathrm{mm}$, which reflects the stroke of the P joint. Second, when a limb is in the $\mathrm{U}_{\mathrm{v}} \mathrm{PS}$ configuration, angle $\psi_{i}(i=1,2,3)$ between the first rotational axis of vA joint and P joint axis shown in Fig. 4 should be in a range of $30 \sim 150$ deg to avoid link interference. Finally, rotational angle of spherical joints denoted by $\varphi_{i}(i=1,2,3)$ should not exceed $45 \mathrm{deg}$. The architectural parameters of the RPM are specified as $a=100 \mathrm{~mm}$ and $b=50 \mathrm{~mm}$. More specifically, detailed constraints of the workspaces for the four configurations of the RPM are listed in Table.3.

Table.3 Constraints of the workspaces

\begin{tabular}{cc}
\hline RPM configurations & Constraints \\
\hline $3 \mathrm{U}_{\mathrm{v}} \mathrm{PS}$ & $80 \mathrm{~mm} \leq d_{1}, d_{2}, d_{3} \leq 220 \mathrm{~mm}, 30 \mathrm{deg} \leq \psi_{1}, \psi_{2}, \psi_{3} \leq 150 \mathrm{deg}, 0 \leq \varphi_{1}, \varphi_{2}, \varphi_{3} \leq 45 \mathrm{deg}$ \\
$2 \mathrm{U}_{\mathrm{v}} \mathrm{PS}-1 \mathrm{R}_{\mathrm{v}} \mathrm{PS}$ & $80 \mathrm{~mm} \leq d_{1}, d_{2}, d_{3} \leq 220 \mathrm{~mm}, 30 \mathrm{deg} \leq \psi_{2}, \psi_{3} \leq 150 \mathrm{deg}, 0 \leq \varphi_{1}, \varphi_{2}, \varphi_{3} \leq 45 \mathrm{deg}$ \\
$1 \mathrm{U}_{\mathrm{v}} \mathrm{PS}-2 \mathrm{R}_{\mathrm{v}} \mathrm{PS}$ & $80 \mathrm{~mm} \leq d_{1}, d_{2}, d_{3} \leq 220 \mathrm{~mm}, 30 \mathrm{deg} \leq \psi_{3} \leq 150 \mathrm{deg}, 0 \leq \varphi_{1}, \varphi_{2}, \varphi_{3} \leq 45 \mathrm{deg}$ \\
$3 \mathrm{R}_{\mathrm{v}} \mathrm{PS}$ & $80 \mathrm{~mm} \leq d_{1}, d_{2}, d_{3} \leq 220 \mathrm{~mm}, 0 \leq \varphi_{1}, \varphi_{2}, \varphi_{3} \leq 45 \mathrm{deg}$ \\
\hline
\end{tabular}




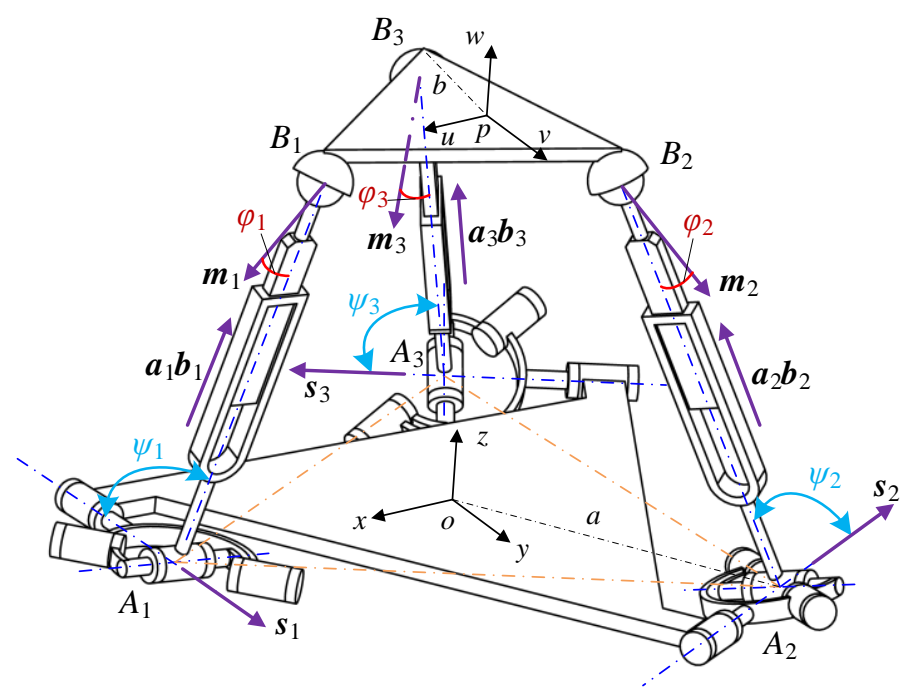

Fig.4 Geometrical model for workspace analysis

Of note is that when the RPM is in the $2 U_{v} P S-1 R_{v} P S$ configuration, limb 1 is assumed to be in the $R_{v} P S$ configuration. While limbs 1 and 2 of the $1 \mathrm{U}_{\mathrm{v}} \mathrm{PS}-2 \mathrm{R}_{\mathrm{v}} \mathrm{PS}$ configuration are $\mathrm{R}_{\mathrm{v}} \mathrm{PS}$ limbs.

In Table.3, $d_{i}(i=1,2,3)$ is the length of vector $\boldsymbol{A}_{i} \boldsymbol{B}_{i}$, and can be identified using Eqs.(7-9) given all the output parameters. $\psi_{i}$ $(i=1,2,3)$ is the angle between vectors $\boldsymbol{s}_{i}$ and $\boldsymbol{a}_{i} \boldsymbol{b}_{i}$, where $\boldsymbol{s}_{i}$ is a unit vector along the first rotational axis of vA joint in limb $i$, and $\boldsymbol{a}_{i} \boldsymbol{b}_{i}$ is a normalized vector of vector $\boldsymbol{A}_{i} \boldsymbol{B}_{i} . \varphi_{i}(i=1,2,3)$ is the angle between vectors $\boldsymbol{m}_{i}$ and $\boldsymbol{a}_{i} \boldsymbol{b}_{i}$, where $\boldsymbol{m}_{i}$ is a unit vector fixed to the moving platform that represents the center line of the spherical joint's socket. Detailed expressions for those vectors expressed in the global coordinate system are given by

$$
\begin{gathered}
\boldsymbol{s}_{1}=[0,1,0]^{\mathrm{T}}, \boldsymbol{s}_{2}=\left[-\frac{\sqrt{3}}{2},-\frac{1}{2}, 0\right]^{\mathrm{T}}, \boldsymbol{s}_{3}=\left[\frac{\sqrt{3}}{2},-\frac{1}{2}, 0\right]^{\mathrm{T}} \\
\boldsymbol{a}_{1} \boldsymbol{b}_{1}=\frac{\boldsymbol{A}_{1} \boldsymbol{B}_{1}}{\left|\boldsymbol{A}_{1} \boldsymbol{B}_{1}\right|}, \boldsymbol{a}_{2} \boldsymbol{b}_{2}=\frac{\boldsymbol{A}_{2} \boldsymbol{B}_{2}}{\left|\boldsymbol{A}_{2} \boldsymbol{B}_{2}\right|}, \boldsymbol{a}_{3} \boldsymbol{b}_{3}=\frac{\boldsymbol{A}_{3} \boldsymbol{B}_{3}}{\left|\boldsymbol{A}_{3} \boldsymbol{B}_{3}\right|} \\
\boldsymbol{m}_{1}={ }^{G} \boldsymbol{R}_{L} \cdot{ }^{L} \boldsymbol{m}_{1}, \boldsymbol{m}_{2}={ }^{G} \boldsymbol{R}_{L} \cdot \boldsymbol{R}_{z}\left(\frac{2 \pi}{3}\right) \cdot{ }^{L} \boldsymbol{m}_{1}, \boldsymbol{m}_{3}={ }^{G} \boldsymbol{R}_{L} \cdot \boldsymbol{R}_{z}\left(\frac{4 \pi}{3}\right) \cdot{ }^{L} \boldsymbol{m}_{1}
\end{gathered}
$$

in which ${ }^{L} \boldsymbol{m}_{1}=\left[-\frac{1}{2}, 0,-\frac{\sqrt{3}}{2}\right]^{\mathrm{T}}$. It is decided by the structural design of the moving platform, which implies that the angle between vector $\boldsymbol{m}_{1}$ and axis $u$ is $60 \mathrm{deg} . \boldsymbol{m}_{1}, \boldsymbol{m}_{2}$, and $\boldsymbol{m}_{3}$ are symmetrically located on the moving platform.

Angles $\psi_{i}(i=1,2,3)$ and $\varphi_{i}(i=1,2,3)$ can be given by

$$
\begin{gathered}
\psi_{1}=\arccos \left(-\boldsymbol{s}_{1} \cdot \boldsymbol{a}_{1} \boldsymbol{b}_{1}\right), \psi_{2}=\arccos \left(-\boldsymbol{s}_{2} \cdot \boldsymbol{a}_{2} \boldsymbol{b}_{2}\right), \psi_{3}=\arccos \left(-\boldsymbol{s}_{3} \cdot \boldsymbol{a}_{3} \boldsymbol{b}_{3}\right) \\
\varphi_{1}=\arccos \left(-\boldsymbol{m}_{1} \cdot \boldsymbol{a}_{1} \boldsymbol{b}_{1}\right), \varphi_{2}=\arccos \left(-\boldsymbol{m}_{2} \cdot \boldsymbol{a}_{2} \boldsymbol{b}_{2}\right), \varphi_{3}=\arccos \left(-\boldsymbol{m}_{3} \cdot \boldsymbol{a}_{3} \boldsymbol{b}_{3}\right)
\end{gathered}
$$

In workspace identification, inverse solutions will be used. First, a search region that should be larger than the real workspace is identified, which is discretized into numerous points. Each point corresponds to a group of output parameters, based on which $d_{i}$, $\psi_{i}$ and $\varphi_{i}$ can be obtained using Eqs.(7-9) and (25-26). Then Table.3 is employed to select points that satisfy the constraints. The selected points finally form the workspace.

Using this search method, the workspaces of the RPM in different configurations are numerically determined as in Figs. 5-8. Fig. 5(a) shows the translation workspace with orientation angles equal to zero. Fig. 5(b) shows the orientation workspace with the platform located at a central position $(x=0, y=0, z=150 \mathrm{~mm})$. Fig. 6 is the orientation workspace of the $2 \mathrm{U}_{\mathrm{v}} \mathrm{PS}-1 \mathrm{R}_{\mathrm{v}} \mathrm{PS}$ configuration with $x=0$ and $z=150 \mathrm{~mm}$. Fig.7 is the orientation workspace of the $1 \mathrm{U}_{\mathrm{v}} \mathrm{PS}-2 \mathrm{R}_{\mathrm{v}} \mathrm{PS}$ configuration with $z=150 \mathrm{~mm}$. Fig. 8 shows the workspace of the $3 \mathrm{R}_{\mathrm{v}} \mathrm{PS}$ configuration.

Generally, orientation workspaces of parallel mechanisms are limited because of their multi-closed loop structures. While we can find from above workspaces that the RPM has large orientation workspace in all configurations. Range of $\beta$ and $\gamma$ are more than $\pm 30 \mathrm{deg}$, and that of $\alpha$ are more than $\pm 45 \mathrm{deg}$. Of note is that for PMs in many applications, performance associated with rotational motion is crucial. Therefore, orientation workspaces of the RPM are concerned here, and the flowing analysis will focus on rotational motion. 


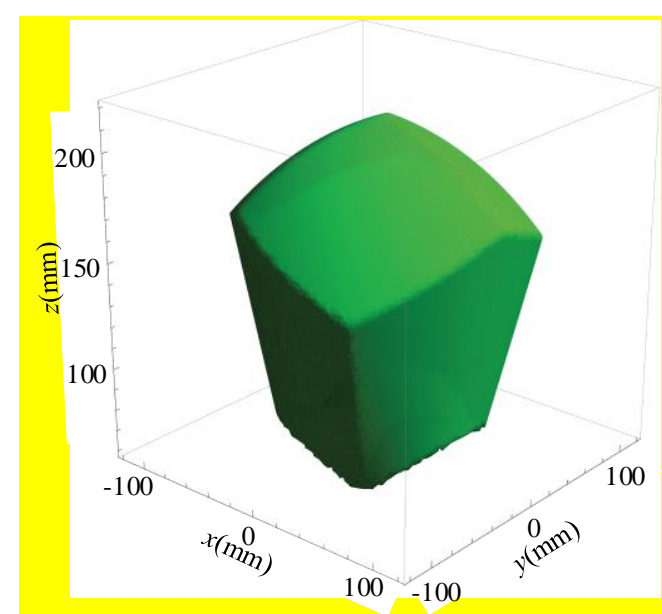

(a)

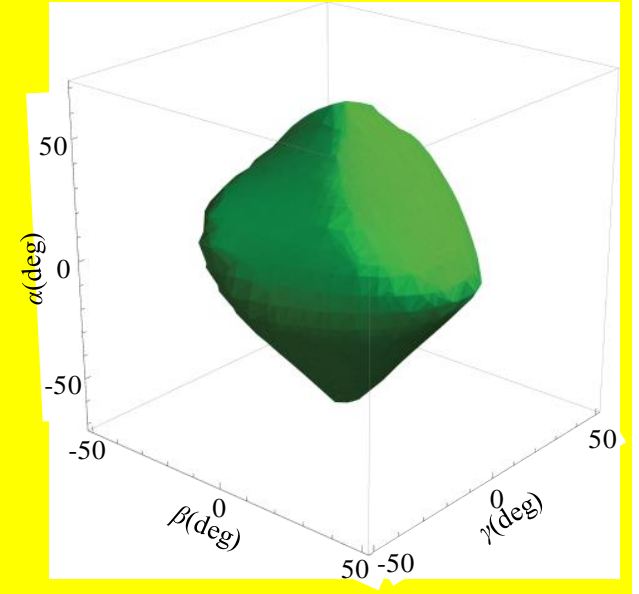

(b)

Fig.5 Workspace of the $3 \mathrm{U}_{\mathrm{v}} \mathrm{PS}$ configuration: (a) translation workspace: $\alpha=\beta=\gamma=0$, (b) orientation workspace: $x=0, y=0, z=150 \mathrm{~mm}$

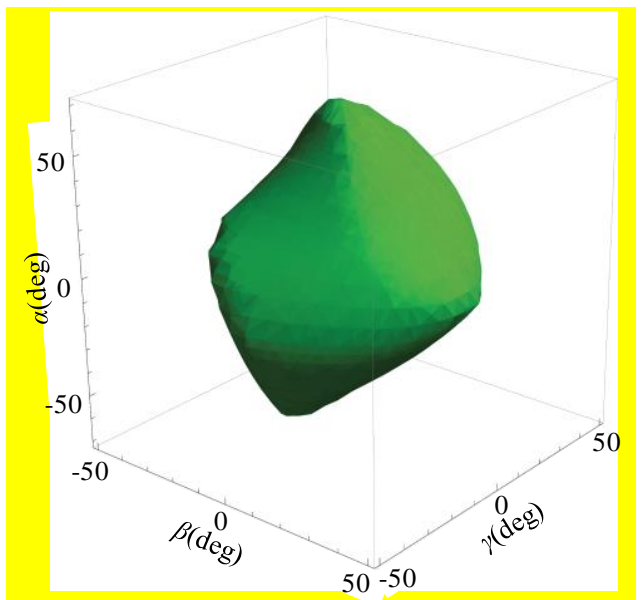

Fig.6 Orientation workspace of the $2 \mathrm{U}_{\mathrm{v}} \mathrm{PS}-1 \mathrm{R}_{\mathrm{v}} \mathrm{PS}$ configuration: $x=0, z=150 \mathrm{~mm}$

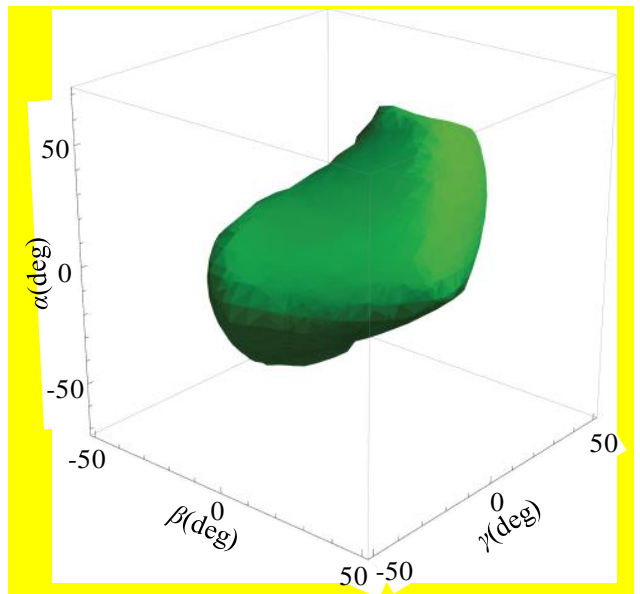

Fig.7 Orientation workspace of the $1 \mathrm{U}_{\mathrm{v}} \mathrm{PS}-2 \mathrm{R}_{\mathrm{v}} \mathrm{PS}$ configuration: $z=150 \mathrm{~mm}$ 


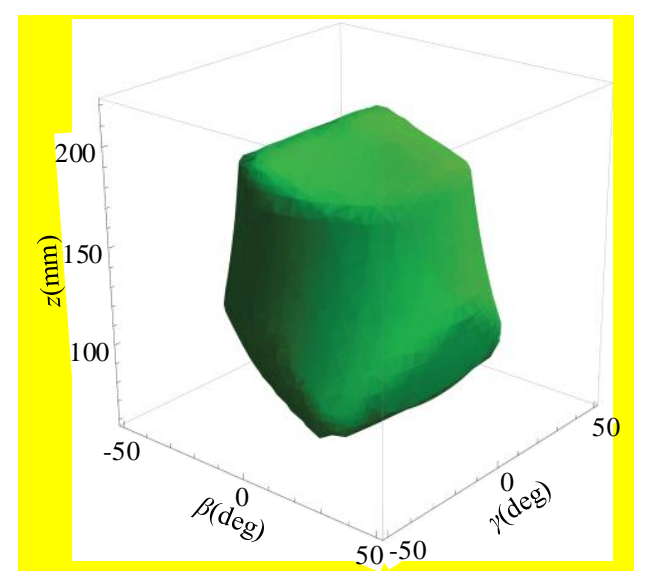

Fig.8 Workspace of the $3 \mathrm{R}_{\mathrm{v}} \mathrm{PS}$ configuration

\subsection{Jacobian and singularity}

Jacobian matrix of a parallel mechanism represents the mapping between the joint input rates and the moving platform output velocity. It is useful in singularity analysis and performance evaluation. For the RPM having four mobility configurations here, it would be better to establish a unified Jacobian model that covers all the configurations. A screw theory based method [33] that is efficient in modeling full Jacobian matrix for parallel mechanisms is used here.

Express the angular velocity of the moving platform with respect to the global coordinate system using a vector $\boldsymbol{w}$, and the linear velocity of a point in the moving platform that is instantaneously coincident with the origin of the reference coordinate system using a vector $\boldsymbol{V}_{p}$. The instantaneous twist of the moving platform can be expressed by $\boldsymbol{S}_{p}=\left[\boldsymbol{w}^{\mathrm{T}} \boldsymbol{v}_{p}^{\mathrm{T}}\right]^{\mathrm{T}}$, which should be a linear combination of twists in limb $i$ as

$$
\begin{array}{cc}
\boldsymbol{S}_{p}=\dot{\theta}_{i 1} \boldsymbol{S}_{i 1}+\dot{\theta}_{i 2} \boldsymbol{S}_{i 2}+\dot{d}_{i} \boldsymbol{S}_{i 3}+\dot{\theta}_{i 4} \boldsymbol{S}_{i 4}+\dot{\theta}_{i 5} \boldsymbol{S}_{i 5}+\dot{\theta}_{i 6} \boldsymbol{S}_{i 6} & \text { for limb } i \text { in U US configuration } \\
\boldsymbol{S}_{p}=\dot{\theta}_{i 1} \boldsymbol{S}_{i 1}+\dot{d}_{i} \boldsymbol{S}_{i 3}+\dot{\theta}_{i 4} \boldsymbol{S}_{i 4}+\dot{\theta}_{i 5} \boldsymbol{S}_{i 5}+\dot{\theta}_{i 6} \boldsymbol{S}_{i 6} & \text { for limb } i \text { in R RS configuration }
\end{array}
$$

where $\boldsymbol{S}_{i 1}$ and $\boldsymbol{S}_{i 2}$ are unit screws of joints $\mathrm{R}_{1}$ and $\mathrm{R}_{3}$ of vA joint in limb $i$, respectively, $\boldsymbol{S}_{i 3}$ is the unit screw of the prismatic joint, and $\boldsymbol{S}_{i 4}, \boldsymbol{S}_{i 5}, \boldsymbol{S}_{i 6}$ are unit screws associated with the spherical joint, as shown in Fig. 9. $\dot{\theta}_{i 1}, \dot{\theta}_{i 2}, \dot{d}_{i}, \dot{\theta}_{i 4}, \dot{\theta}_{i 5}$ and $\dot{\theta}_{i 6}$ are intensities of those twists. Expressions for joints screws in Eqs.(27-28) are

$$
\begin{aligned}
& \boldsymbol{S}_{i 1}=\left[\begin{array}{ll}
\boldsymbol{s}_{i}^{\mathrm{T}} & \boldsymbol{A}_{i}^{\mathrm{T}} \times \boldsymbol{s}_{i}^{\mathrm{T}}
\end{array}\right]^{\mathrm{T}} \\
& \boldsymbol{S}_{i 2}=\left[\begin{array}{ll}
\boldsymbol{n}_{i}^{\mathrm{T}} & \boldsymbol{A}_{i}^{\mathrm{T}} \times \boldsymbol{n}_{i}^{\mathrm{T}}
\end{array}\right]^{\mathrm{T}} \\
& \boldsymbol{S}_{i 3}=\left[\begin{array}{ll}
\mathbf{0}^{\mathrm{T}} & \boldsymbol{a}_{i} \boldsymbol{b}_{i}^{\mathrm{T}}
\end{array}\right]^{\mathrm{T}} \quad(i=1,2,3) \\
& \boldsymbol{S}_{i 4}=\left[\begin{array}{ll}
\boldsymbol{x}^{\mathrm{T}} & \boldsymbol{B}_{i}^{\mathrm{T}} \times \boldsymbol{x}^{\mathrm{T}}
\end{array}\right]^{\mathrm{T}} \quad \\
& \boldsymbol{S}_{i 5}=\left[\begin{array}{ll}
\boldsymbol{y}^{\mathrm{T}} & \boldsymbol{B}_{i}^{\mathrm{T}} \times \boldsymbol{y}^{\mathrm{T}}
\end{array}\right]^{\mathrm{T}} \\
& \boldsymbol{S}_{i 6}=\left[\begin{array}{ll}
\boldsymbol{z}^{\mathrm{T}} & \boldsymbol{B}_{i}^{\mathrm{T}} \times \boldsymbol{z}^{\mathrm{T}}
\end{array}\right]^{\mathrm{T}}
\end{aligned}
$$

in which $\boldsymbol{x}, \boldsymbol{y}$ and $\boldsymbol{z}$ are unit vectors along axes $x, y$ and $z$, respectively.

When limb $i$ is in U $\mathrm{vS}$ configuration, $\boldsymbol{S}_{i 1}$ and $\boldsymbol{S}_{i 3}$ are actuated joint screws. A transmission wrench screw [34], $\boldsymbol{S}_{r i 1}=\left[\begin{array}{ll}\boldsymbol{n}_{i}^{\mathrm{T}} & \boldsymbol{B}_{i}^{\mathrm{T}} \times \boldsymbol{n}_{i}^{\mathrm{T}}\end{array}\right]^{\mathrm{T}}$ that is reciprocal to joint screws $\boldsymbol{S}_{i 2}, \boldsymbol{S}_{i 3}, \boldsymbol{S}_{i 4}, \boldsymbol{S}_{i 5}, \boldsymbol{S}_{i 6}$ except for $\boldsymbol{S}_{i 1}$ can be found, which is a force wrench passing through the center of the spherical joint and parallel to the axis of $\boldsymbol{S}_{i 2}$. This force is an actuated force transmitted from the actuation to the moving platform. Taking the orthogonal product of both sides of Eq. (27) with $\boldsymbol{S}_{r i 1}$ yields

$$
\boldsymbol{S}_{r i 1}^{T} \boldsymbol{S}_{p}=\boldsymbol{S}_{r i 1}^{T} \boldsymbol{S}_{i 1} \dot{\theta}_{i 1}
$$

Similarly, a transmission wrench screw, $\boldsymbol{S}_{r i 2}=\left[\begin{array}{llllll}\boldsymbol{a}_{i} \boldsymbol{b}_{i}^{\mathrm{T}} \boldsymbol{A}_{i}^{\mathrm{T}} \times \boldsymbol{a}_{i} \boldsymbol{b}_{i}^{\mathrm{T}}\end{array}\right]^{\mathrm{T}}$, that is reciprocal to joint screws $\quad \boldsymbol{S}_{i 1}, \boldsymbol{S}_{i 2}, \quad \boldsymbol{S}_{i 4}, \boldsymbol{S}_{i 5}$, $\boldsymbol{S}_{i 6}$ except for $\boldsymbol{S}_{i 3}$ can be found, which is a force wrench passing through the centers of the vA joint and the spherical joint in limb $i$. Taking the orthogonal product of both sides of Eq. (27) with $\boldsymbol{S}_{r i 2}$ yields

$$
\boldsymbol{S}_{r i 2}^{T} \boldsymbol{S}_{p}=\boldsymbol{S}_{r i 2}^{T} \boldsymbol{S}_{i 3} \dot{d}_{i}
$$




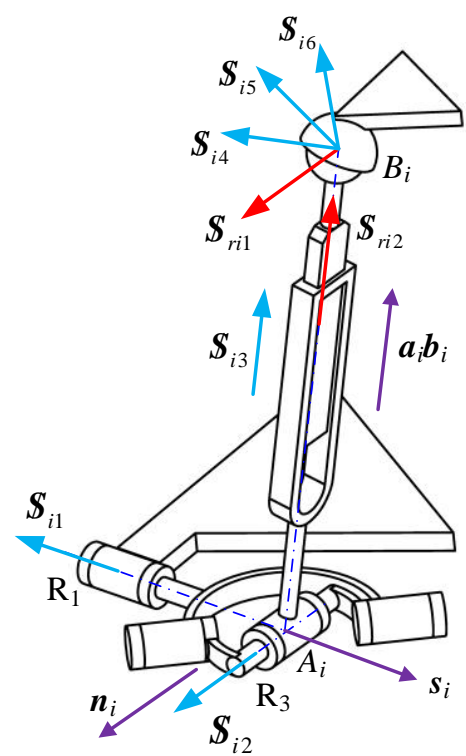

(a)

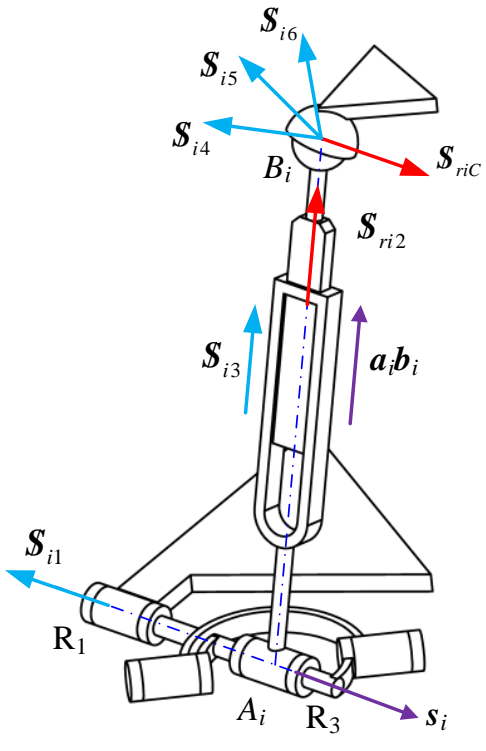

(b)

Fig.9 Screws in limb $i$ : (a) $\mathrm{U}_{\mathrm{v}} \mathrm{PS}$ configuration, (b) $\mathrm{R}_{\mathrm{v}} \mathrm{PS}$ configuration

Limb $i$ in $\mathrm{R}_{\mathrm{v}} \mathrm{PS}$ configuration has five DOFs. A constraint screw $\boldsymbol{S}_{r i c}=\left[\boldsymbol{s}_{i}^{\mathrm{T}} \boldsymbol{B}_{i}^{\mathrm{T}} \times \boldsymbol{s}_{i}^{\mathrm{T}}\right]^{\mathrm{T}}$ that is reciprocal to all the joint screws in Eq.(28) can be found. It is a constraint force exerted on the moving platform by the limb, which satisfies the following equation:

$$
\boldsymbol{S}_{r i c}^{T} \boldsymbol{S}_{p}=0
$$

In $R_{v} P S$ configuration, $\boldsymbol{S}_{i 3}$ is the actuated screws. Its corresponding transmission wrench screw is still $\boldsymbol{S}_{r i 2}$, and Eq. (31) is also applicable to the $\mathrm{R}_{\mathrm{v}} \mathrm{PS}$ configuration.

According to the above analysis, a matrix form equation can be established for the RPM as

in which the undefined variables are given in Table.4.

$$
\left[\begin{array}{c}
\boldsymbol{S}_{r 12}^{T} \\
\boldsymbol{S}_{r 22}^{T} \\
\boldsymbol{S}_{r 32}^{T} \\
\boldsymbol{S}_{1}^{T} \\
\boldsymbol{S}_{2} \\
\boldsymbol{S}_{3}
\end{array}\right] \boldsymbol{S}_{p}=\left[\begin{array}{cccccc}
\boldsymbol{S}_{r 12}^{T} \boldsymbol{S}_{13} & 0 & 0 & 0 & 0 & 0 \\
0 & \boldsymbol{S}_{r 22}^{T} \boldsymbol{S}_{23} & 0 & 0 & 0 & 0 \\
0 & 0 & \boldsymbol{S}_{r 32}^{T} \boldsymbol{S}_{33} & 0 & 0 & 0 \\
0 & 0 & 0 & \boldsymbol{S}_{4} & 0 & 0 \\
0 & 0 & 0 & 0 & \boldsymbol{S}_{5} & 0 \\
0 & 0 & 0 & 0 & 0 & \boldsymbol{S}_{6}
\end{array}\right]\left[\begin{array}{c}
\dot{d}_{1} \\
\dot{d}_{2} \\
\dot{d}_{3} \\
\dot{q}_{1} \\
\dot{q}_{2} \\
\dot{q}_{3}
\end{array}\right]
$$

Table.4 Variables in Eq. (33)

\begin{tabular}{cccccccccc}
\hline RPM configurations & $\boldsymbol{S}_{1}$ & $\boldsymbol{S}_{2}$ & $\boldsymbol{S}_{3}$ & $\boldsymbol{S}_{4}$ & $\boldsymbol{S}_{5}$ & $\boldsymbol{S}_{6}$ & $\dot{q}_{1}$ & $\dot{q}_{2}$ & $\dot{q}_{3}$ \\
\hline $3 \mathrm{U}_{\mathrm{v}} \mathrm{PS}$ & $\boldsymbol{S}_{r 11}^{T}$ & $\boldsymbol{S}_{r 21}^{T}$ & $\boldsymbol{S}_{r 31}^{T}$ & $\boldsymbol{S}_{r 11}^{T} \boldsymbol{S}_{11}$ & $\boldsymbol{S}_{r 21}^{T} \boldsymbol{S}_{21}$ & $\boldsymbol{S}_{r 31}^{T} \boldsymbol{S}_{31}$ & $\dot{\theta}_{11}$ & $\dot{\theta}_{21}$ & $\dot{\theta}_{31}$ \\
$2 \mathrm{U}_{\mathrm{v}} \mathrm{PS}-1 \mathrm{R}_{\mathrm{v}} \mathrm{PS}$ & $\boldsymbol{S}_{r 1 C}^{T}$ & $\boldsymbol{S}_{r 21}^{T}$ & $\boldsymbol{S}_{r 31}^{T}$ & 0 & $\boldsymbol{S}_{r 21}^{T} \boldsymbol{S}_{21}$ & $\boldsymbol{S}_{r 31}^{T} \boldsymbol{S}_{31}$ & 0 & $\dot{\theta}_{21}$ & $\dot{\theta}_{31}$ \\
$1 \mathrm{U}_{\mathrm{v}} \mathrm{PS}-2 \mathrm{R}_{\mathrm{v}} \mathrm{PS}$ & $\boldsymbol{S}_{r 1 C}^{T}$ & $\boldsymbol{S}_{r 2 C}^{T}$ & $\boldsymbol{S}_{r 31}^{T}$ & 0 & 0 & $\boldsymbol{S}_{r 31}^{T} \boldsymbol{S}_{31}$ & 0 & 0 & $\dot{\theta}_{31}$ \\
$3 \mathrm{R}_{\mathrm{v}} \mathrm{PS}$ & $\boldsymbol{S}_{r 1 C}^{T}$ & $\boldsymbol{S}_{r 2 C}^{T}$ & $\boldsymbol{S}_{r 3 C}^{T}$ & 0 & 0 & 0 & 0 & 0 & 0 \\
\hline
\end{tabular}

Eq. (33) is the unified Jacobin model for the RPM in its four configurations. It can be written in a general form as

$$
\boldsymbol{J}_{x} \boldsymbol{S}_{p}=\boldsymbol{J}_{q} \dot{\boldsymbol{q}}
$$

in which 


$$
\boldsymbol{J}_{x}=\left[\begin{array}{c}
\boldsymbol{S}_{r 12}^{T} \\
\boldsymbol{S}_{r 22}^{T} \\
\boldsymbol{S}_{r 32}^{T} \\
\boldsymbol{S}_{1} \\
\boldsymbol{S}_{2} \\
\boldsymbol{S}_{3}
\end{array}\right], \quad \boldsymbol{J}_{q}=\left[\begin{array}{cccccc}
\boldsymbol{S}_{r 12}^{T} \boldsymbol{S}_{13} & 0 & 0 & 0 & 0 & 0 \\
0 & \boldsymbol{S}_{r 22}^{T} \boldsymbol{S}_{23} & 0 & 0 & 0 & 0 \\
0 & 0 & \boldsymbol{S}_{r 32}^{T} \boldsymbol{S}_{33} & 0 & 0 & 0 \\
0 & 0 & 0 & \boldsymbol{S}_{4} & 0 & 0 \\
0 & 0 & 0 & 0 & \boldsymbol{S}_{5} & 0 \\
0 & 0 & 0 & 0 & 0 & \boldsymbol{S}_{6}
\end{array}\right], \quad \dot{\boldsymbol{q}}=\left[\begin{array}{c}
\dot{d}_{1} \\
\dot{d}_{2} \\
\dot{d}_{3} \\
\dot{q}_{1} \\
\dot{q}_{2} \\
\dot{q}_{3}
\end{array}\right]
$$

It is convenient to conduct singularity analysis for a parallel mechanism based on its Jacobina matrix. There are generally three kinds of singularities [35] in parallel mechanisms, i.e., forward kinematic singularity, inverse kinematic singularity and combined singularity. Forward kinematic singularity, also called type 2 singularity [36], is generally inside the workspace and is commonly studied [37]. Inverse kinematic singularity and combined singularity are not concerned here since they occur at the workspace boundary.

Forward kinematic singularity occurs when $\operatorname{det}\left(\boldsymbol{J}_{x}\right)=0$, which can be easily identified through numerical calculation. Singular curves of the RPM in different configurations are obtained as in Fig. 10-13. It can be concluded that when the RPM is in the $3 \mathrm{U}_{\mathrm{v}} \mathrm{PS}$ configuration, its translation workspace with orientation angles equal to 0 is divided into six subspaces by singular surfaces, and its orientation workspace in the central position is divided into two subspaces, which indicates performances in those workspaces will not be good. Similarly, performance in orientation workspace of the $1 \mathrm{U}_{\mathrm{v}} \mathrm{PS}-2 \mathrm{R}_{\mathrm{v}} \mathrm{PS}$ configuration is poor. On the other hand, performances of the $2 U_{v} P S-1 R_{v} P S$ configuration and $3 R_{v} P S$ configuration would be satisfactory since the singular surfaces are far from the workspace center.

Furthermore, singular configurations corresponding to selected points on the singular curves are displayed in Fig. 10-13. Those singularities can be explained by reduced-rank of $\boldsymbol{J} x$. For example, Fig. 10(c) shows the 3 UvPS configuration with output parameters $\alpha=\beta=\gamma=0, x=0, y=0$, and $z=150 \mathrm{~mm}$. In that pose, the three transmission wrench screws $\boldsymbol{\$}_{r 11}, \boldsymbol{\$}_{r 21}$, and $\boldsymbol{\$}_{r 31}$ intersect at a common point $E$, and the three transmission wrench screws $\$_{r 12}, \$_{r 22}$, and $\$_{r 32}$ intersect at the other common point $F$. Those six screws are dependent, which cannot totally restrict the DOFs of the mechanism. A rotational DOF around line $E F$ is out of control.

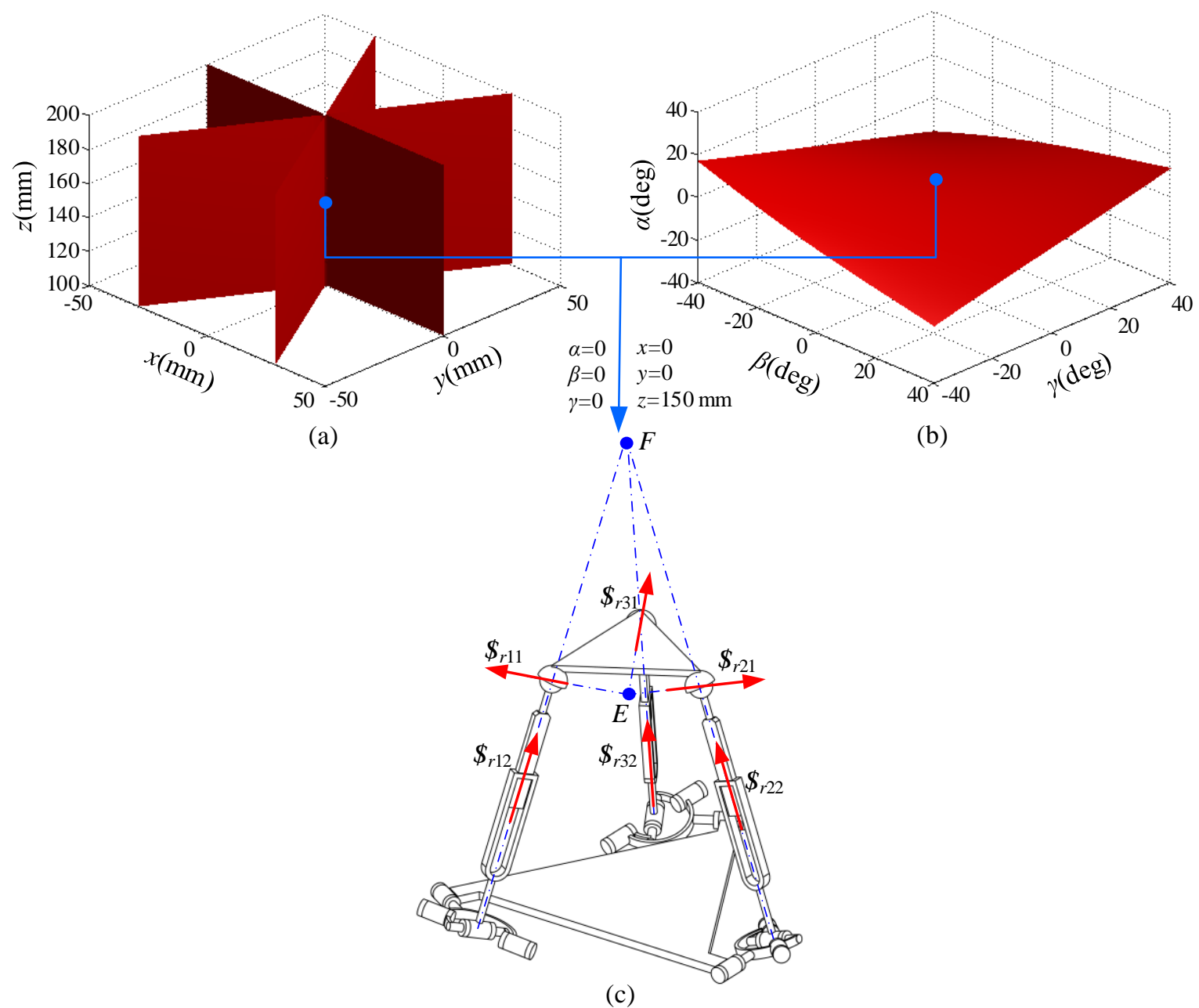

Fig.10 Singularity of the $3 \mathrm{U}_{\mathrm{v}} \mathrm{PS}$ configuration: (a) singular curve in translation workspace: $\alpha=\beta=\gamma=0$, (b) singular curve in orientation workspace: $x=0, y=0, z=150 \mathrm{~mm}$, (c) singular configuration 


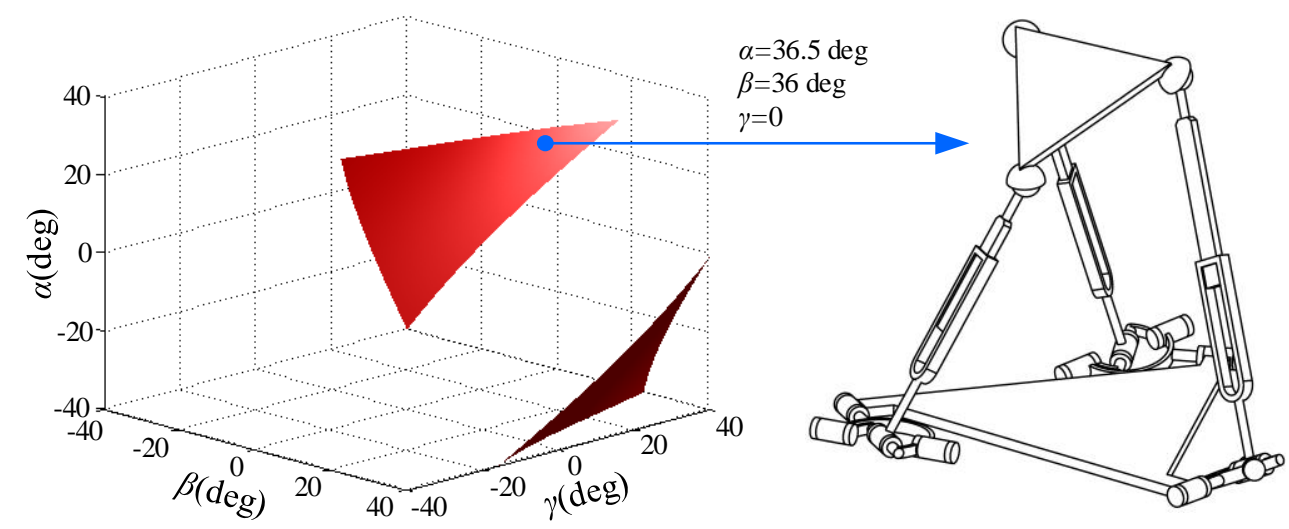

(a)

(b)

Fig.11 Singularity of the $2 \mathrm{U}_{\mathrm{v}} \mathrm{PS}-1 \mathrm{R}_{\mathrm{v}} \mathrm{PS}$ configuration: (a) singular curve with $x=0$ and $z=150 \mathrm{~mm}$, (b) singular configuration

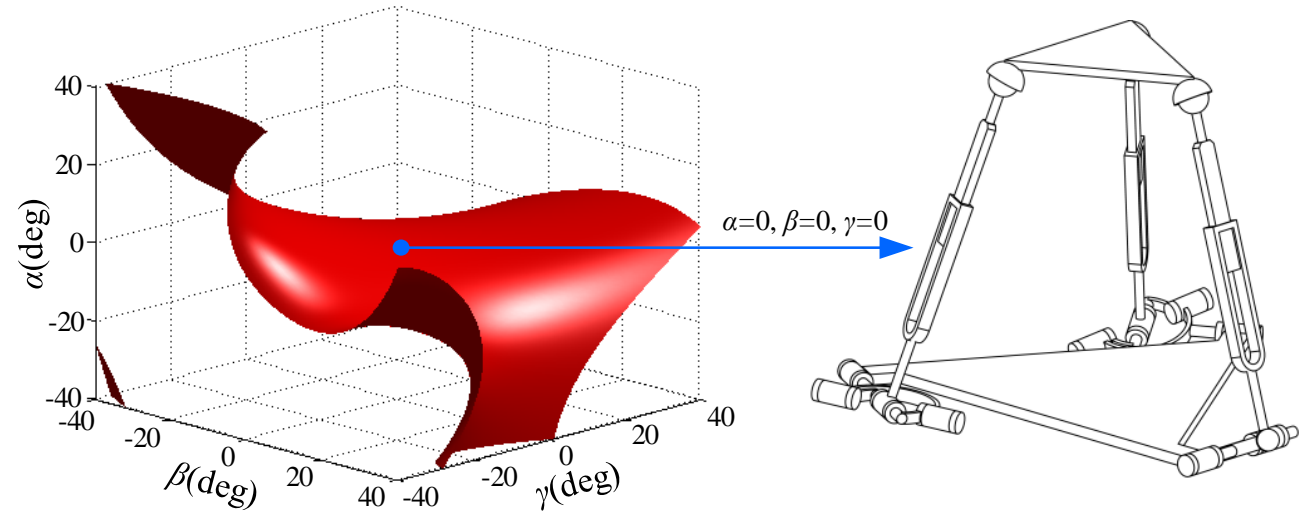

(a)

(b)

Fig.12 Singularity of the $1 \mathrm{U}_{\mathrm{v}} \mathrm{PS}-2 \mathrm{R}_{\mathrm{v}} \mathrm{PS}$ configuration: (a) singular curve with $z=150 \mathrm{~mm}$, (b) singular configuration

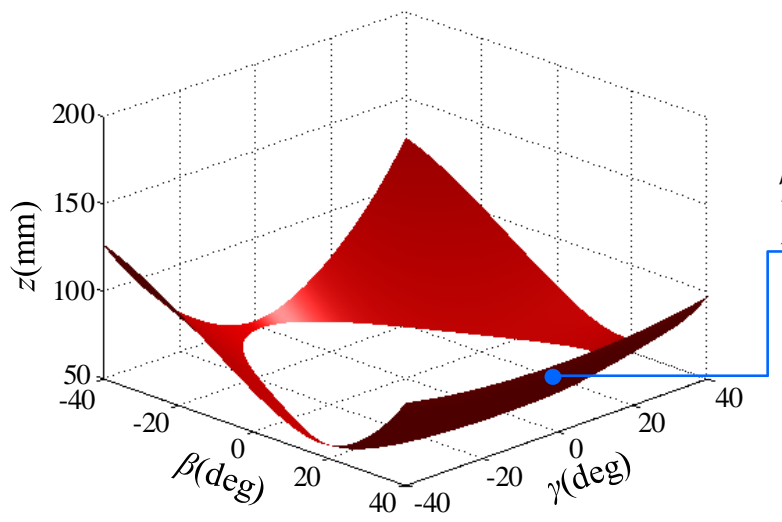

(a)

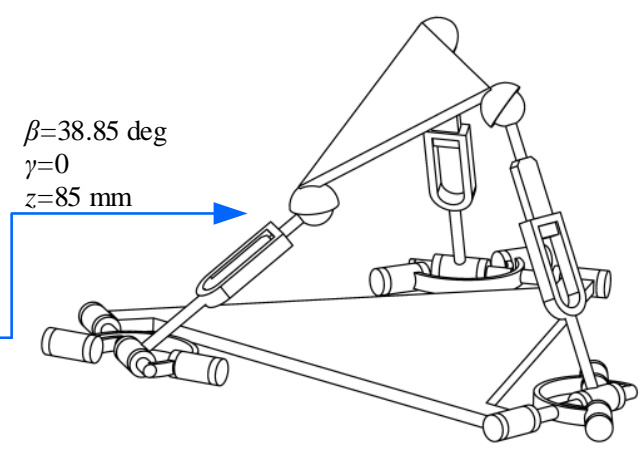

(b)

Fig.13 Singularity of the $3 \mathrm{R}_{\mathrm{v}} \mathrm{PS}$ configuration: (a) singular curve, (b) singular configuration

\section{Performance analysis and optimization}

Singularity analysis only indicates whether the mechanism is singular or not, but cannot measure how the kinematics performance is for a nonsingular condition. To know the performance of the RPM in the workspace, performance analysis should be carried out. Among various methods for performance measure of parallel mechanisms, the method of motion/force transmission [34, 38-39] is dimensionless, independent with reference frame, physically clear, and has been widely used. It will be utilized here to establish a unified evaluation model for the RPM. 


\subsection{Local transmission index}

The motion/force transmission method defines input transmission index, output transmission index, and local transmission index. According to the definition, when limb $i$ is in the $U_{v} P S$ configuration, it has two input transmission indices. The one for the actuated revolute joint $\mathrm{R}_{1}$ is given by

$$
\lambda_{i 1}=\frac{\left|\$_{i 1} \circ \$_{r i 1}\right|}{\left|\$_{i 1} \circ \$_{r i 1}\right|_{\max }}
$$

The other one for the actuated prismatic joint $\mathrm{P}$ is given by

$$
\lambda_{i 2}=\frac{\left|\$_{i 3} \circ \$_{r i 2}\right|}{\left|\$_{i 3} \circ \$_{r i 2}\right|_{\max }}
$$

Limb $i$ in the $\mathrm{R}_{\mathrm{v}} \mathrm{PS}$ configuration has only one input transmission index that is $\lambda_{i 2}$ since only the $\mathrm{P}$ joint is actuated. An input index $\lambda_{i}$ for limb $i$ covering $\mathrm{U}_{\mathrm{v}} \mathrm{PS}$ configuration and $\mathrm{R}_{\mathrm{v}} \mathrm{PS}$ configuration can be defined as

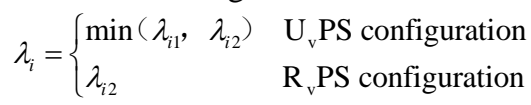

Similarly, limb $i$ in the $\mathrm{U}_{\mathrm{v}} \mathrm{PS}$ configuration has two output transmission indices. One for transmission wrench $\$_{r i 1}$ and the other for transmission wrench $\$_{r i 2}$. They are

$$
\begin{aligned}
& \eta_{i 1}=\frac{\left|\$_{\mathrm{O} i 1} \circ \$_{r i 1}\right|}{\left|\$_{\mathrm{O} i 1} \circ \$_{r i 1}\right|_{\max }} \\
& \eta_{i 2}=\frac{\left|\$_{\mathrm{O} i 2} \circ \$_{r i 2}\right|}{\left|\$_{\mathrm{O} i 2} \circ \$_{r i 2}\right|_{\max }}
\end{aligned}
$$

where $\$_{\mathrm{O} i 1}\left(\$_{\mathrm{O} i 2}\right)$ is the motion screw of the moving platform with all the actuated joints in the RPM locked except the one associated with $\$_{r i 1}\left(\$_{r i 2}\right)$, which can be derived using reciprocal screw theory [34].

Limb $i$ in the $\mathrm{R}_{\mathrm{v}} \mathrm{PS}$ configuration has only one output transmission index $\eta_{i 2}$ because only the $\mathrm{P}$ joint is actuated. An output index $\eta_{i}$ for limb $i$ covering $\mathrm{U}_{\mathrm{v}} \mathrm{PS}$ configuration and $\mathrm{R}_{\mathrm{v}} \mathrm{PS}$ configuration can be defined as

$$
\eta_{i}= \begin{cases}\min \left(\eta_{i 1},\right. & \left.\eta_{i 2}\right) \\ \eta_{i 2} & \mathrm{U}_{\mathrm{v}} \mathrm{PS} \text { configuration } \\ \mathrm{R}_{\mathrm{v}} \mathrm{PS} \text { configuration }\end{cases}
$$

When the input transmission index and output transmission index are identified, an overall transmission index can be given by

$$
\Gamma=\min \left\{\lambda_{i}, \eta_{i}\right\}(i=1,2,3)
$$

Since the transmission index $\Gamma$ will be different when the mechanism's position and orientation parameters are different, it is referred to as the local transmission index (LTI), which ranges from 0 to 1 [34]. Large LTI indicates good motion/force transmission performance and small LTI means the mechanism is close to singularity. The LTI distribution of the RPM in different configuration are sketched as in Figs. 14-18. We can find that the LTI distributions conform to the singularities in Figs. 10-13. Figs. 14 and 15 show that the $3 \mathrm{U}_{\mathrm{v}} \mathrm{PS}$ configuration has poor performance when tilt angle $\alpha$ equals 0 , but relatively good performance when $\alpha$ is not 0 (such as $\pi / 6$ ), which can be explained by different singularity conditions. The $2 \mathrm{U}_{\mathrm{v}} \mathrm{PS}-1 \mathrm{R}_{\mathrm{v}} \mathrm{PS}$ configuration and $3 \mathrm{R}_{\mathrm{v}} \mathrm{PS}$ configuration have good motion/force transmission performance since LTI values in most of the workspace, especially in the central region, are relatively high. While the performance of the $1 \mathrm{U}_{\mathrm{v}} \mathrm{PS}-2 \mathrm{R}_{\mathrm{v}} \mathrm{PS}$ configuration is poor because of the singularities inside the workspace as in Fig. 12.

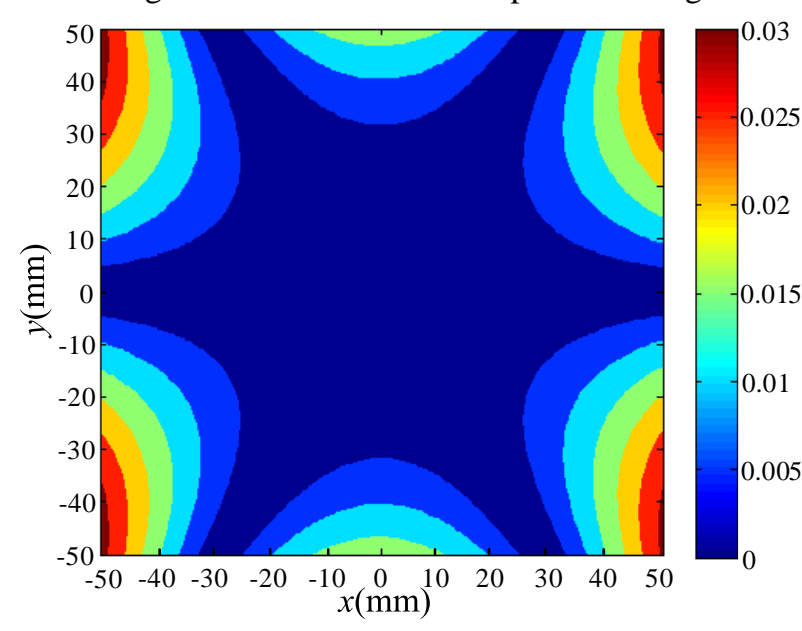

(a)

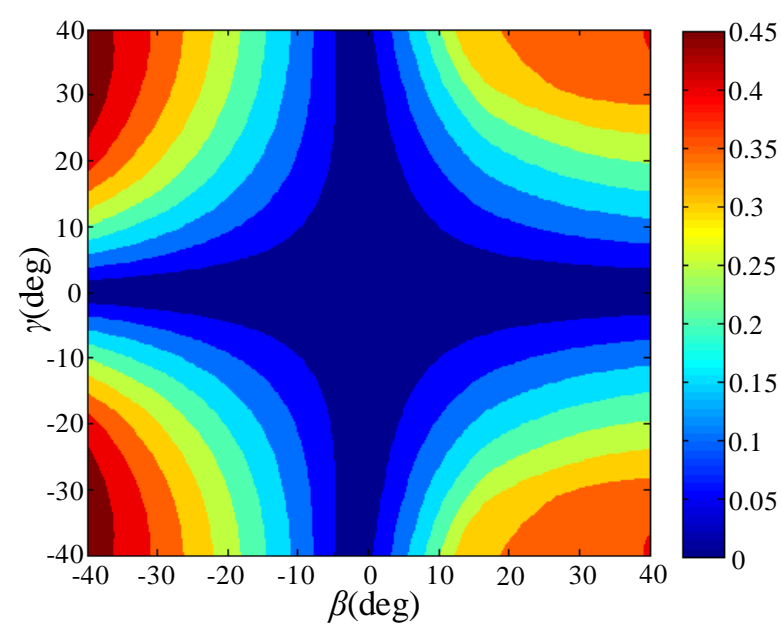

(b)

Fig.14 LTI distribution of the $3 \mathrm{U}_{\mathrm{v}} \mathrm{PS}$ configuration: (a) $\alpha=\beta=\gamma=0, z=150 \mathrm{~mm}$, (b) $\alpha=0, x=0, y=0, z=150 \mathrm{~mm}$ 


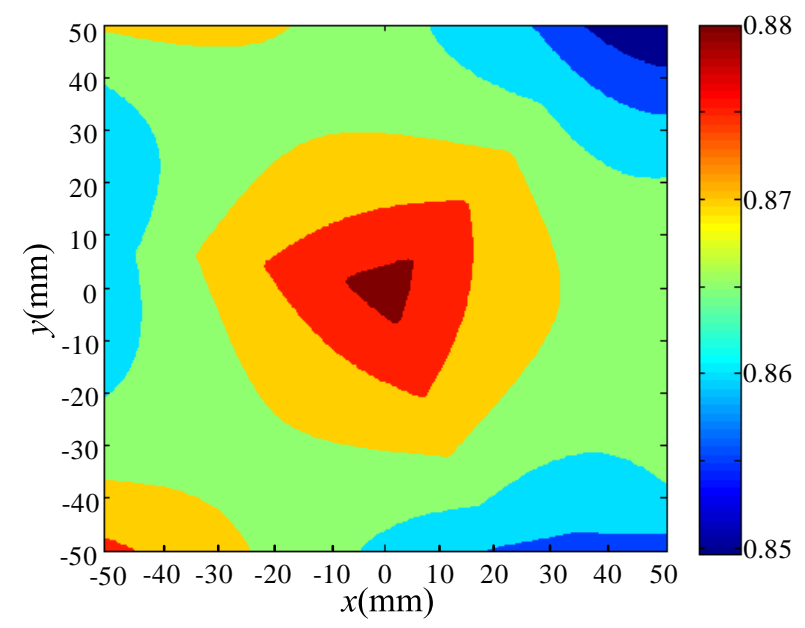

(a)

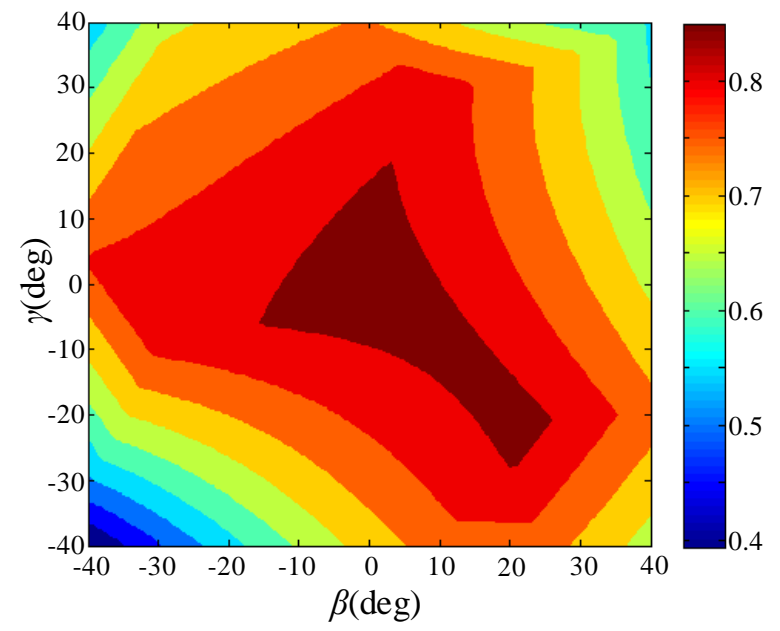

(b)

Fig.15 LTI distribution of the $3 \mathrm{U}_{\mathrm{v}} \mathrm{PS}$ configuration: (a) $\alpha=\pi / 6, \beta=\gamma=0, z=150 \mathrm{~mm}$ (b) $\alpha=\pi / 6, x=0, y=0, z=150 \mathrm{~mm}$

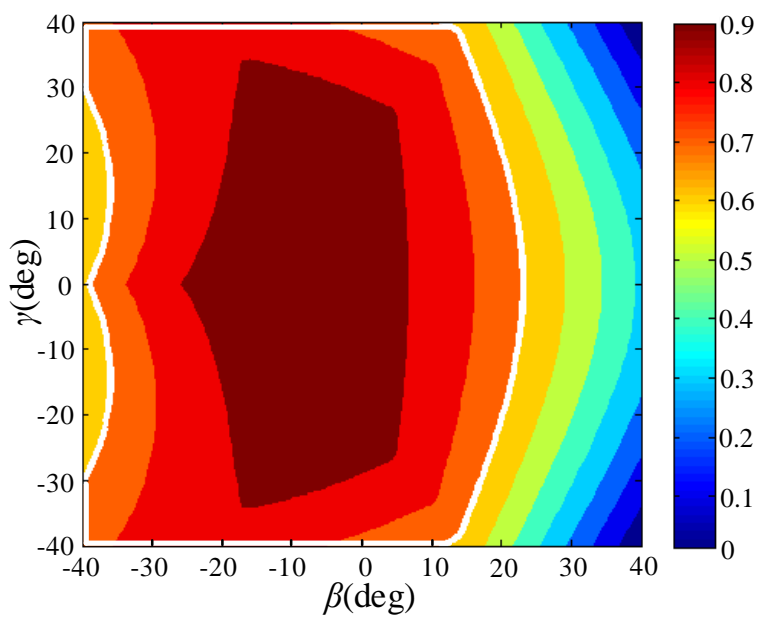

Fig.16 LTI distribution of the $2 \mathrm{U}_{\mathrm{v}} \mathrm{PS}-1 \mathrm{R}_{\mathrm{v}} \mathrm{PS}$ configuration: $\alpha=0, x=0, z=150 \mathrm{~mm}$

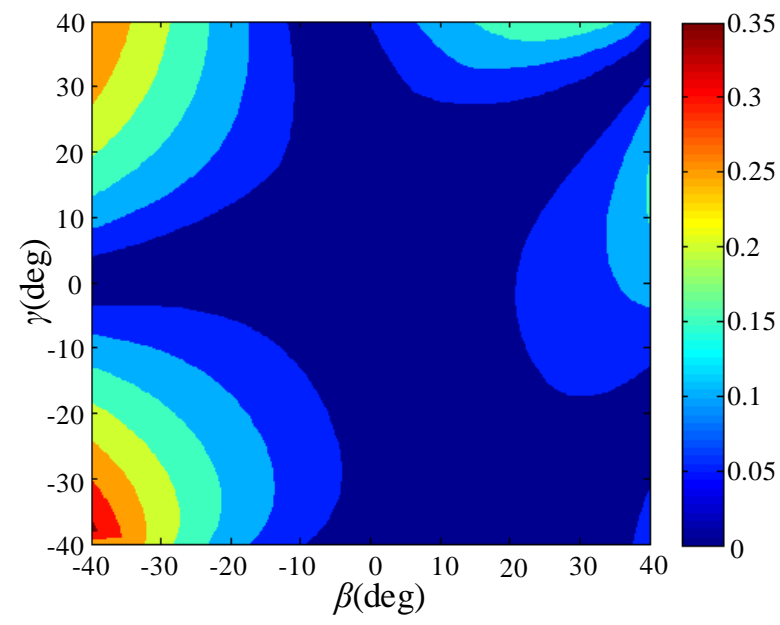

Fig.17 LTI distribution of the $1 \mathrm{U}_{\mathrm{v}} \mathrm{PS}-2 \mathrm{R}_{\mathrm{v}} \mathrm{PS}$ configuration: $\alpha=0, z=150 \mathrm{~mm}$ 


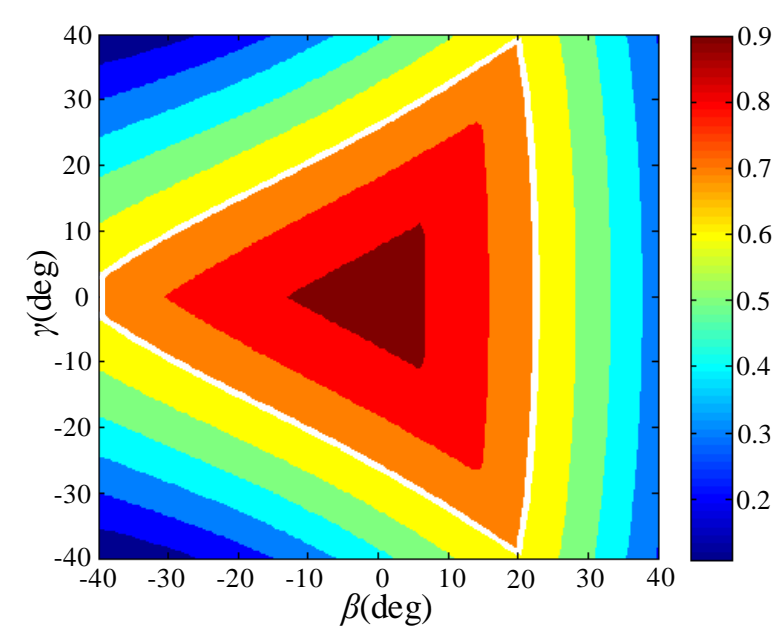

Fig.18 LTI distribution of the $3 \mathrm{R}_{\mathrm{v}} \mathrm{PS}$ configuration: $z=150 \mathrm{~mm}$

\subsection{Global transmission index and optimization}

LTI represents motion/force transmission performance of the RPM in a single point of the workspace. To measure the global performance throughout the prescribed workspace, a global performance index is required. Define the region with $\Gamma \geq 0.7$ [34] as good transmission workspace, the ratio of good transmission workspace to the prescribed workspace is given by

$$
\sigma=\frac{\mathrm{GTW}}{\mathrm{PW}}
$$

where GTW represents the area of the good transmission workspace, and PW represents the area of the whole prescribed workspace. $\sigma$ ranges from 0 to 1 , and large $\sigma$ means good global transmission performance.

For a same prescribed workspace, a mechanism with different dimensions will have different global transmission performance, which means $\sigma$ can be used in performance optimization. Here we select the $2 \mathrm{U}_{\mathrm{v}} \mathrm{PS}-1 \mathrm{R}_{\mathrm{v}} \mathrm{PS}$ configuration, the $3 \mathrm{R}_{\mathrm{v}} \mathrm{PS}$ configuration as examples, and analyze their global transmission performance with different dimensional parameters $a$ and $b$. The results in a design space of $a \in[80 \mathrm{~mm}, 120 \mathrm{~mm}]$ and $b \in[40 \mathrm{~mm}, 60 \mathrm{~mm}]$ are shown in Figs 19 .

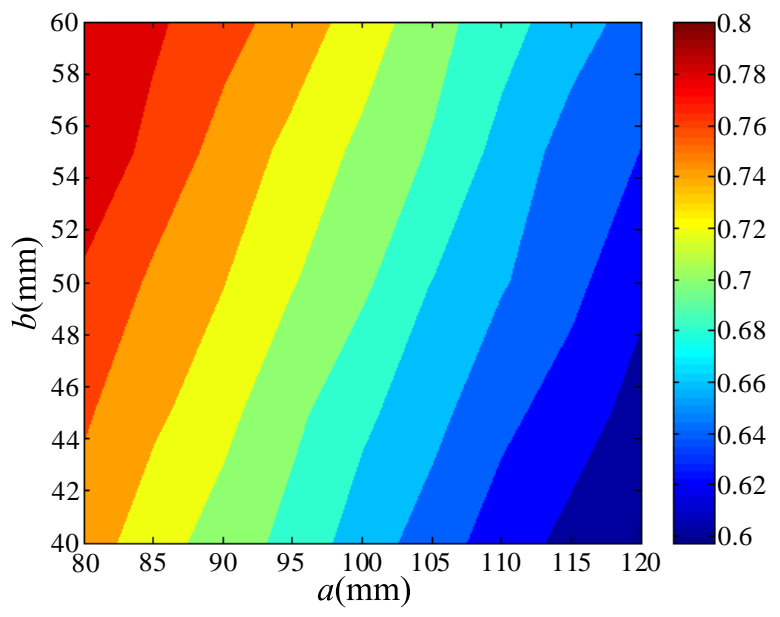

(a)

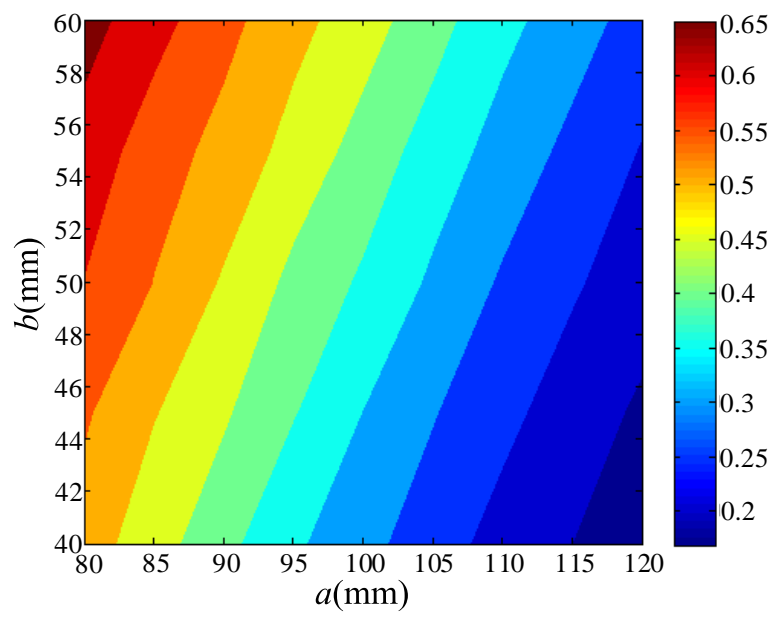

(b)

Fig.19 Global transmission performance: (a) $2 \mathrm{U}_{\mathrm{v}} \mathrm{PS}-1 \mathrm{R}_{\mathrm{v}} \mathrm{PS}$ configuration, (b) $3 \mathrm{R}_{\mathrm{v}} \mathrm{PS}$ configuration

Fig. 19 shows that variations of parameters $a$ and $b$ have similar influence on global performances of the $2 \mathrm{U}_{\mathrm{v}} \mathrm{PS}-1 \mathrm{R}_{\mathrm{v}} \mathrm{PS}$ configuration and the $3 \mathrm{R}_{\mathrm{v}} \mathrm{PS}$ configuration. Smaller $a$ and larger $b$ result in better global performance in the prescribed workspace. Fig. 16 and Fig. 18 show the LTI distributions with $a=100 \mathrm{~mm}$ and $b=50 \mathrm{~mm}$. For comparison purpose, LTI distributions of the $2 \mathrm{U}_{\mathrm{v}} \mathrm{PS}-1 \mathrm{R}_{\mathrm{v}} \mathrm{PS}$ configuration and the $3 \mathrm{R}_{\mathrm{v}} \mathrm{PS}$ configuration with $a=80 \mathrm{~mm}$ and $b=60 \mathrm{~mm}$ are plotted in Fig. 20. Figs. 16, 18, and 20 show that compared with the initial dimensional parameters, good transmission workspaces (the regions encircled by white lines) of those two configurations with optimized parameters are larger, which means the global performances are improved and the optimization method is effective. 


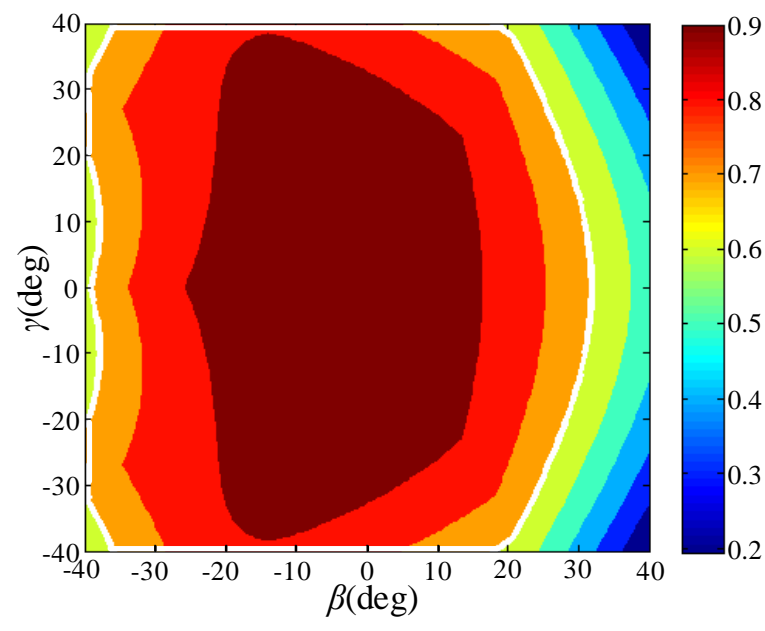

(a)

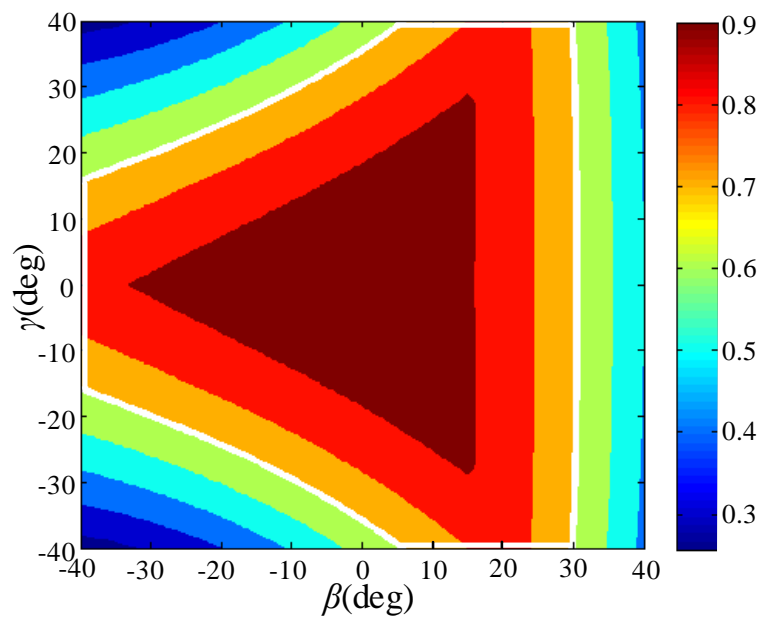

(b)

Fig.20 LTI distribution: (a) $2 \mathrm{U}_{\mathrm{v}} \mathrm{PS}-1 \mathrm{R}_{\mathrm{v}} \mathrm{PS}$ configuration, $\alpha=0, x=0, z=150 \mathrm{~mm}$, (b) $3 \mathrm{R}_{\mathrm{v}} \mathrm{PS}$ configuration, $z=150 \mathrm{~mm}$

\section{Conclusion}

This paper constructed a new RPM with three kinematic limbs. Each limb contains a metamorphic variable-axis (vA) joint with changeable phases. With the vA joints evolved into different phases, the kinematic limbs change their configurations and constraints, which lead to reconfiguration of the RPM. The RPM has four mobility configurations including 6-DOF, 5-DOF, 4DOF and 3-DOF configurations. Kinematic modeling and workspace analysis for the RPM were revealed. A unified Jacobian model that covers all the configurations was established based on the methods using screw theory. Singularities were obtained and sketched as surfaces in workspace corresponding to different configurations. Local transmission index and global transmission index were established in performance analysis and optimization of the RPM. The results show that the 6-DOF configuration with a tilt angle, the 5-DOF configuration, and the 3-DOF configuration have relatively good motion/force transmission performance, which can be further improved through dimensional optimization. A reconfigurable machine center was conceived by integrating the RPM with a X-Y gantry. Its reconfigurability and adaptability enable users to select a preferable working mode according to the task requirements.

\section{Acknowledgments}

The work is supported by the National Natural Science Foundation of China (NSFC) under Grant No. 51705465 and Zhejiang Provincial Natural Science Foundation of China under Grant No. LQ19E050015, Engineering and Physical Sciences Research Council (EPSRC) National Centre for Nuclear Robotics under Grant No. EP/R02572X/1 and School of Engineering and Materials Science, Queen Mary University of London.

\section{References}

[1] Pierrot F, Reynaud C, Fournier A. DELTA: a simple and efficient parallel robot[J]. Robotica, 1990, 8(2): 105-109.

[2] Hennes N. Ecospeed-an innovative machinery concept for high performance 5 axis machining of large structural components in aircraft engineering[C]//3rd Chemnitz Parallel Kinematic Seminar. 2002: 763-774.

[3] Jin Y, Bi Z M, Liu H T, et al. Kinematic analysis and dimensional synthesis of exechon parallel kinematic machine for large volume machining[J]. Journal of Mechanisms and Robotics, 2015, 7(4): 041004.

[4] Siciliano B. The Tricept robot: Inverse kinematics, manipulability analysis and closed-loop direct kinematics algorithm[J]. Robotica, 1999, 17(4): 437-445.

[5] Xie F, Liu X J. Design and development of a high-speed and high-rotation robot with four identical arms and a single platform[J]. Journal of Mechanisms and Robotics, 2015, 7(4): 041015.

[6] Dong C, Liu H, Yue W, et al. Stiffness modeling and analysis of a novel 5-DOF hybrid robot[J]. Mechanism and Machine Theory, 2018, 125: 80-93.

[7] Liu H, Dai J. An approach to carton-folding trajectory planning using dual robotic fingers[J]. Robotics and Autonomous Systems, 2003, 42(1): 47-63.

[8] Kong X, Gosselin C M, Richard P L. Type synthesis of parallel mechanisms with multiple operation modes[J]. Journal of Mechanical Design, 2007, 129(6): 595-601.

[9] Kong X. Type synthesis of 3-DOF parallel manipulators with both a planar operation mode and a spatial translational operation mode[J]. Journal of Mechanisms and Robotics, 2013, 5(4): 041015.

[10] Kong X, Yu J. Type synthesis of two-degrees-of-freedom 3-4R parallel mechanisms with both spherical translation mode and sphere-on-sphere rolling mode[J]. Journal of Mechanisms and Robotics, 2015, 7(4): 041018. 
[11] Kong X. Reconfiguration analysis of a 3-DOF parallel mechanism using Euler parameter quaternions and algebraic geometry method[J]. Mechanism and Machine Theory, 2014, 74: 188-201.

[12] Li Q, Hervé J M. Parallel mechanisms with bifurcation of Schoenflies motion[J]. IEEE Transactions on Robotics, 2009, 25(1): 158-164.

[13] Gogu G. Maximally regular T2R1-type parallel manipulators with bifurcated spatial motion[J]. Journal of Mechanisms and Robotics, 2011, 3(1): 011010.

[14] Gan D, Dai J S, Liao Q. Mobility change in two types of metamorphic parallel mechanisms[J]. Journal of Mechanisms and Robotics, 2009, 1(4): 041007.

[15] Gan D, Dai J S, Liao Q. Constraint analysis on mobility change of a novel metamorphic parallel mechanism[J]. Mechanism and Machine Theory, 2010, 45(12): 1864-1876.

[16] Gan D, Dai J S, Caldwell D G. Constraint-based limb synthesis and mobility-change-aimed mechanism construction[J]. Journal of Mechanical Design, 2011, 133(5): 051001.

[17] Gan D, Dias J, Seneviratne L. Unified kinematics and optimal design of a 3rRPS metamorphic parallel mechanism with a reconfigurable revolute joint[J]. Mechanism and Machine Theory, 2016, 96: 239-254.

[18] Zhang K, Dai J S, Fang Y. Topology and constraint analysis of phase change in the metamorphic chain and its evolved mechanism[J]. Journal of Mechanical Design, 2010, 132(12): 121001.

[19] Zhang K, Dai J S, Fang Y. Geometric constraint and mobility variation of two 3SvPSv metamorphic parallel mechanisms[J]. Journal of Mechanical Design, 2013, 135(1): 011001.

[20] Carbonari L, Callegari M, Palmieri G, et al. A new class of reconfigurable parallel kinematic machines[J]. Mechanism and Machine Theory, 2014, 79: 173-183.

[21] Palpacelli M C, Carbonari L, Palmieri G, et al. Analysis and design of a reconfigurable 3-DoF parallel manipulator for multimodal tasks[J]. IEEE/Asme Transactions on Mechatronics, 2014, 20(4): 1975-1985.

[22] Carbonari L, Costa D, Palmieri G, et al. Reconfigurability analysis of a class of parallel kinematics machines[J]. Journal of Mechanisms and Robotics, 2019, 11(2): 021002.

[23] Daniel Finistauri A. Reconfiguration analysis of a fully reconfigurable parallel robot[J]. Journal of Mechanisms and Robotics, 2013, 5(4).

[24] Ye W, Fang Y, Zhang K, et al. A new family of reconfigurable parallel mechanisms with diamond kinematotropic chain[J]. Mechanism and Machine Theory, 2014, 74: 1-9.

[25] Ye W, Fang Y F, Guo S. Reconfigurable parallel mechanisms with planar five-bar metamorphic linkages[J]. Science China Technological Sciences, 2014, 57(1): 210-218.

[26] Ye W, Fang Y, Zhang K, et al. Mobility variation of a family of metamorphic parallel mechanisms with reconfigurable hybrid limbs[J]. Robotics and Computer-Integrated Manufacturing, 2016, 41: 145-162.

[27] Zlatanov D, Bonev I A, Gosselin C M. Constraint singularities as C-space singularities[M]//Advances in robot kinematics. Springer, Dordrecht, 2002: 183-192.

[28] Gan D, Dai J S, Dias J, et al. Reconfigurability and unified kinematics modeling of a 3rTPS metamorphic parallel mechanism with perpendicular constraint screws[J]. Robotics and Computer-Integrated Manufacturing, 2013, 29(4): 121128.

[29] Gan D, Dai J S, Dias J, et al. Unified kinematics and singularity analysis of a metamorphic parallel mechanism with bifurcated motion[J]. Journal of Mechanisms and Robotics, 2013, 5(3): 031004.

[30] Nurahmi L, Gan D. Reconfiguration of a 3-(rR) PS Metamorphic Parallel Mechanism Based on Complete Workspace and Operation Mode Analysis[J]. Journal of Mechanisms and Robotics, 2020, 12(1).

[31] Carbonari L, Corinaldi D, Palmieri G, et al. Kinematics of a Novel 3-URU Reconfigurable Parallel Robot[C]//2018 International Conference on Reconfigurable Mechanisms and Robots (ReMAR). IEEE, 2018: 1-7.

[32] Huang Z, Fang Y. Motion characteristics and rotational axis analysis of three DOF parallel robot mechanisms[C]//1995 IEEE International Conference on Systems, Man and Cybernetics. Intelligent Systems for the 21st Century. IEEE, 1995, 1: 67-71.

[33] Joshi S A, Tsai L W. Jacobian analysis of limited-DOF parallel manipulators[J]. Journal of Mechanical Design, 2002, 124(2): 254-258.

[34] Wang J, Wu C, Liu X J. Performance evaluation of parallel manipulators: Motion/force transmissibility and its index[J]. Mechanism and Machine Theory, 2010, 45(10): 1462-1476.

[35] Tsai L W. Robot analysis: the mechanics of serial and parallel manipulators[M]. John Wiley \& Sons, 1999.

[36] Gosselin C, Angeles J. Singularity analysis of closed-loop kinematic chains[J]. IEEE transactions on robotics and automation, 1990, 6(3): 281-290.

[37] Gan D, Dai J S, Dias J, et al. Forward kinematics solution distribution and analytic singularity-free workspace of linearactuated symmetrical spherical parallel manipulators[J]. Journal of Mechanisms and Robotics, 2015, 7(4): 041007.

[38] Xie F, Liu X J, Wang J. A 3-DOF parallel manufacturing module and its kinematic optimization[J]. Robotics and ComputerIntegrated Manufacturing, 2012, 28(3): 334-343.

[39] Xie F G, Liu X J, Zhang H, et al. Design and experimental study of the SPKM165, a five-axis serial-parallel kinematic milling machine[J]. Science China Technological Sciences, 2011, 54(5): 1193-1205. 